\title{
Higher torsion in the Abelianization of the full Bianchi groups
}

\author{
Alexander D. Rahm
}

\begin{abstract}
Denote by $\mathbb{Q}(\sqrt{-m})$, with $m$ a square-free positive integer, an imaginary quadratic number field, and by $\mathcal{O}_{-m}$ its ring of integers. The Bianchi groups are the groups $\mathrm{SL}_{2}\left(\mathcal{O}_{-m}\right)$. In the literature, so far there have been no examples of $p$-torsion in the integral homology of the full Bianchi groups, for $p$ a prime greater than the order of elements of finite order in the Bianchi group, which is at most 6 . However, extending the scope of the computations, we can observe examples of torsion in the integral homology of the quotient space, at prime numbers as high as for instance $p=80737$ at the discriminant -1747 .

Supplementary materials are available with this article.
\end{abstract}

\section{Introduction}

The Bianchi groups $\Gamma:=\mathrm{SL}_{2}\left(\mathcal{O}_{-m}\right)$ may be considered as a key to the study of a larger class of groups, the Kleinian groups, which date back to work of Henri Poincaré [17]. In fact, each non-co-compact arithmetic Kleinian group is commensurable with some Bianchi group [14]. A wealth of information on the Bianchi groups can be found in the monographs $[\mathbf{8}, \mathbf{9}, \mathbf{1 4}]$. In the literature, so far there have been no examples of $p$-torsion in the integral homology of the full Bianchi groups, for $p$ a prime greater than the order of elements of finite order in the Bianchi group (a recent survey of relevant calculations has been given in [29]). In fact, the numerical studies that have been made so far, were carried out in the range where the quotient space of hyperbolic 3 -space $\mathcal{H}$ by the Bianchi group is often homotopy equivalent to a wedge sum of 2-spheres, 2-tori and Möbius bands [33].

We make use of Serre's decomposition $[\mathbf{3 1}]$ of the homology group $H_{1}(\Gamma \backslash \mathcal{H} ; \mathbb{Z})$ into the direct sum of the free Abelian group with one generator for each element of the class group of $\mathcal{O}_{-m}$ and the group $\mathrm{H}_{1}^{\text {cusp }}(\Gamma \backslash \mathcal{H} ; \mathbb{Z})$ computed in Tables 1 and 2. The first computations of $\mathrm{H}_{1}(\Gamma ; \mathbb{Z}) \supset \mathrm{H}_{1}(\Gamma \backslash \mathcal{H} ; \mathbb{Z})$ by Swan $[32]$ were on a range of Bianchi groups with vanishing cuspidal homology $\mathrm{H}_{1}^{\text {cusp }}(\Gamma \backslash \mathcal{H} ; \mathbb{Z})$. The first example where $\mathrm{H}_{1}^{\text {cusp }}(\Gamma \backslash \mathcal{H} ; \mathbb{Z})$ is non-zero, occurred in an unpublished calculation of Mennicke. Swan's manual computations of group presentations have been extended on the computer by Riley [25]; and later Vogtmann [33] and Scheutzow $[\mathbf{2 6}]$ systematically computed $\mathrm{H}_{1}^{\text {cusp }}(\Gamma \backslash \mathcal{H} ; \mathbb{Q})$ for a large range of Bianchi groups. But they were still in the range where $\Gamma \backslash \mathcal{H}$ admits no homological torsion. Aranes [1] has computed cell complexes for the Bianchi groups for all $m \leqslant 100$, and Yasaki [35] has obtained $\mathrm{GL}_{2}\left(\mathcal{O}_{-m}\right)$-cell complexes (with the Voronoï model) for the same range as well as all cases where $\mathcal{O}$ is of class number 1 or 2 . This includes two cases, $m=74$ and $m=86$, where some 2-torsion appears in $\mathrm{H}_{1}^{\text {cusp }}(\Gamma \backslash \mathcal{H} ; \mathbb{Z})$, but the latter two authors have not yet provided homology computations. When the absolute value of the discriminant becomes greater, torsion in the integral homology of the quotient space appears (see Table 2) at prime numbers as high as for instance 80737 at the discriminant -1747 , whereas the order of elements of finite order in $\mathrm{SL}_{2}\left(\mathcal{O}_{-m}\right)$ is at most 6 . A growth of the torsion in the Abelianization of the Bianchi groups with respect to the covolume can be observed, which is in concordance with the predictions of [3].

Received 18 January 2013; revised 16 May 2013.

2010 Mathematics Subject Classification 11F75 (primary).

Funded by the Irish Research Council. 
We can also observe that the occurring torsion subgroups are quite likely to occur as squares, but this is no general principle, because the discriminant -431 produces a counterexample to this phenomenon.

In order to obtain the results of Tables 1 and 2, in $\S 7$ we fill out Swan's concept [32] and elaborate algorithms to compute a fundamental polyhedron for the action of the Bianchi groups on hyperbolic 3-space. Other algorithms based on the same concept have independently been implemented by Cremona [7] for the five cases where $\mathcal{O}_{-m}$ is Euclidean, and by his students Whitley [34] for the non-Euclidean principal ideal domain cases, Bygott [5] for a case of class number 2 and Lingham [13] (used in [6]) for some cases of class number 3 and, finally, Aranés [1] for arbitrary class numbers. The algorithms presented in $\S 7$ come with an implementation [18] for all Bianchi groups and we make explicit use of the cell complexes it produces (the source code of Bianchi.gp is available as online supplementary material available for download from the publisher's website). The provided implementation [18] has been validated by the project PLUME of the CNRS, and is subject to the certificate C3I of the GENCI and the CPU. Other results obtained with the employed implementation are described in $[\mathbf{1 9}, \mathbf{2 4}]$. On the computing clusters of the Weizmann Institute of Science, this implementation has been applied to establish a database of cell complexes for over 180 Bianchi groups, using over fifty processormonths. This database includes all of the cases of ideal class numbers 3 and 5 , most of the cases of ideal class number 4 and all of the cases of discriminant absolute value bounded by 500 .

A computational advantage is the shortcut that we obtain in $\S 4$ by linking the BorelSerre compactification of the quotient space with Flöge's compactification in a long exact sequence, based on the recent paper [22]. Flöge's compactification admits a computationally easier cell structure, and we can explicitly calculate the equivariant Leray-Serre spectral sequence associated with it. In $\S 5$, we describe how to assemble the homology of the Borel-Serre compactified quotient space and the Farrell cohomology of a Bianchi group to its full group homology with trivial $\mathbb{Z}$-coefficients. Here, we divide by the center of $\mathrm{SL}_{2}\left(\mathcal{O}_{-m}\right)$, consisting of plus and minus the identity matrix, yielding $\operatorname{PSL}_{2}\left(\mathcal{O}_{-m}\right)$. As the center of $\operatorname{SL}_{2}\left(\mathcal{O}_{-m}\right)$ is the kernel of its action on hyperbolic 3 -space, this does not change the quotient space. And for $\Gamma:=\operatorname{PSL}_{2}\left(\mathcal{O}_{-m}\right)$, general formulae for its Farrell cohomology have been given [21] (based on $[20])$.

\subsection{Organization of the paper}

We print in Tables 1 and 2 the isomorphism types of $\mathrm{H}_{1}^{\text {cusp }}(\Gamma \backslash \mathcal{H} ; \mathbb{Z})$ that were obtained. The homology group $\mathrm{H}_{1}(\Gamma \backslash \mathcal{H} ; \mathbb{Z})$ is a direct sum of the former homology group and the free Abelian group with rank the cardinality of the class group of $\mathcal{O}_{-m}$, which we also print. The group homology $\mathrm{H}_{1}(\Gamma ; \mathbb{Z})$ is an extension of $\mathrm{H}_{1}(\Gamma \backslash \mathcal{H} ; \mathbb{Z})$ by a quotient of the Farrell supplement that has been computed and printed in a separate column. In $\S 2$, we define the Bianchi fundamental polyhedron, which induces our cell structure on $\Gamma \backslash \mathcal{H}$. We use it in $\S 3$ to obtain the Flöge cellular complex, which we connect in $\S 4$ to the Borel-Serre compactification of $\Gamma \backslash \mathcal{H}$. Then we proceed to $\mathrm{H}_{1}\left(\mathrm{PSL}_{2}\left(\mathcal{O}_{-m}\right) ; \mathbb{Z}\right)$ in $\S 5$, describe Swan's concept in $\S 6$ and its realization in $\S 7$.

\section{The Bianchi fundamental polyhedron}

Let $m$ be a squarefree positive integer and consider the imaginary quadratic number field $\mathbb{Q}(\sqrt{-m})$ with ring of integers $\mathcal{O}_{-m}$, which we also just denote by $\mathcal{O}$. Consider the familiar action by fractional linear transformations (we give an explicit formula for it in Lemma 22) of the group $\Gamma:=\mathrm{SL}_{2}(\mathcal{O}) \subset \mathrm{GL}_{2}(\mathbb{C})$ on hyperbolic 3-space, for which we will use the upper-half space model $\mathcal{H}$. As a set,

$$
\mathcal{H}=\{(z, \zeta) \in \mathbb{C} \times \mathbb{R} \mid \zeta>0\} .
$$

The Bianchi-Humbert theory $[\mathbf{2}, \mathbf{1 2}]$ gives a fundamental domain for this action. We will start by giving a geometric description of it, and the arguments why it is a fundamental domain. 
TABLE 1. The cuspidal homology $\mathrm{H}_{1}^{\text {cusp }}(\Gamma \backslash \mathcal{H} ; \mathbb{Z})$ for the absolute values of the discriminant $\Delta$ fulfilling $|\Delta|<500$.

\begin{tabular}{|c|c|c|c|c|}
\hline$\Delta$ & $m$ & Class group & $\mathrm{H}_{1}^{\text {cusp }}(\Gamma \backslash \mathcal{H} ; \mathbb{Z})$ & Farrell supplement \\
\hline-7 & 7 & $\{1\}$ & 0 & $\mathbb{Z} / 2$ \\
\hline-8 & 2 & $\{1\}$ & 0 & $\mathbb{Z} / 2 \oplus \mathbb{Z} / 3$ \\
\hline-11 & 11 & $\{1\}$ & 0 & $\mathbb{Z} / 3$ \\
\hline-15 & 15 & $\mathbb{Z} / 2$ & 0 & $\mathbb{Z} / 2 \oplus \mathbb{Z} / 3$ \\
\hline-19 & 19 & $\{1\}$ & 0 & 0 \\
\hline-20 & 5 & $\mathbb{Z} / 2$ & 0 & $(\mathbb{Z} / 2)^{2} \oplus \mathbb{Z} / 3$ \\
\hline-23 & 23 & $\mathbb{Z} / 3$ & 0 & $\mathbb{Z} / 2 \oplus \mathbb{Z} / 3$ \\
\hline-24 & 6 & $\mathbb{Z} / 2$ & 0 & $\mathbb{Z} / 2 \oplus \mathbb{Z} / 3$ \\
\hline-31 & 31 & $\mathbb{Z} / 3$ & 0 & $\mathbb{Z} / 2$ \\
\hline-35 & 35 & $\mathbb{Z} / 2$ & $\mathbb{Z}$ & $\mathbb{Z} / 2 \oplus \mathbb{Z} / 3$ \\
\hline-39 & 39 & $\mathbb{Z} / 4$ & 0 & $\mathbb{Z} / 2 \oplus \mathbb{Z} / 3$ \\
\hline-40 & 10 & $\mathbb{Z} / 2$ & $\mathbb{Z}$ & $(\mathbb{Z} / 2)^{2} \oplus \mathbb{Z} / 3$ \\
\hline-43 & 43 & $\{1\}$ & $\mathbb{Z}$ & 0 \\
\hline-47 & 47 & $\mathbb{Z} / 5$ & 0 & $\mathbb{Z} / 2 \oplus \mathbb{Z} / 3$ \\
\hline-51 & 51 & $\mathbb{Z} / 2$ & $\mathbb{Z}$ & $\mathbb{Z} / 3$ \\
\hline-52 & 13 & $\mathbb{Z} / 2$ & $\mathbb{Z}$ & $(\mathbb{Z} / 2)^{2}$ \\
\hline-55 & 55 & $\mathbb{Z} / 4$ & $\mathbb{Z}$ & $\mathbb{Z} / 2 \oplus \mathbb{Z} / 3$ \\
\hline-56 & 14 & $\mathbb{Z} / 4$ & $\overline{\mathbb{Z}}$ & $(\mathbb{Z} / 2)^{2} \oplus \mathbb{Z} / 3$ \\
\hline-59 & 59 & $\mathbb{Z} / 3$ & $\mathbb{Z}$ & $\mathbb{Z} / 3$ \\
\hline-67 & 67 & $\{1\}$ & $\mathbb{Z}^{2}$ & 0 \\
\hline-68 & 17 & $\mathbb{Z} / 4$ & $\mathbb{Z}$ & $(\mathbb{Z} / 2)^{2} \oplus \mathbb{Z} / 3$ \\
\hline-71 & 71 & $\mathbb{Z} / 7$ & 0 & $\mathbb{Z} / 2 \oplus \mathbb{Z} / 3$ \\
\hline-79 & 79 & $\mathbb{Z} / 5$ & $\mathbb{Z}$ & $(\mathbb{Z} / 2)^{3}$ \\
\hline-83 & 83 & $\mathbb{Z} / 3$ & $\mathbb{Z}^{2}$ & $\mathbb{Z} / 3$ \\
\hline-84 & 21 & $\mathbb{Z} / 2 \times \mathbb{Z} / 2$ & $\mathbb{Z}^{3}$ & $(\mathbb{Z} / 2)^{3} \oplus(\mathbb{Z} / 3)^{2}$ \\
\hline-87 & 87 & $\mathbb{Z} / 6$ & $\mathbb{Z}^{2}$ & $\mathbb{Z} / 2 \oplus \mathbb{Z} / 3$ \\
\hline-88 & 22 & $\mathbb{Z} / 2$ & $\mathbb{Z}^{3}$ & $\mathbb{Z} / 2 \oplus \mathbb{Z} / 3$ \\
\hline-91 & 91 & $\mathbb{Z} / 2$ & $\mathbb{Z}^{3}$ & $\mathbb{Z} / 2$ \\
\hline-95 & 95 & $\mathbb{Z} / 8$ & $\mathbb{Z}$ & $\mathbb{Z} / 2 \oplus \mathbb{Z} / 3$ \\
\hline-103 & 103 & $\mathbb{Z} / 5$ & $\mathbb{Z}^{2}$ & $\mathbb{Z} / 2$ \\
\hline-104 & 26 & $\mathbb{Z} / 6$ & $\mathbb{Z}^{2}$ & $(\mathbb{Z} / 2)^{2} \oplus(\mathbb{Z} / 3)^{2}$ \\
\hline-107 & 107 & $\mathbb{Z} / 3$ & $\mathbb{Z}^{3}$ & $(\mathbb{Z} / 3)^{3}$ \\
\hline-111 & 111 & $\mathbb{Z} / 8$ & $\mathbb{Z}^{2}$ & $\mathbb{Z} / 2 \oplus \mathbb{Z} / 3$ \\
\hline-115 & 115 & $\mathbb{Z} / 2$ & $\mathbb{Z}^{5}$ & $\mathbb{Z} / 2 \oplus \mathbb{Z} / 3$ \\
\hline-116 & 29 & $\mathbb{Z} / 6$ & $\mathbb{Z}^{3}$ & $(\mathbb{Z} / 2)^{2} \oplus \mathbb{Z} / 3$ \\
\hline-119 & 119 & $\mathbb{Z} / 10$ & $\mathbb{Z}$ & $(\mathbb{Z} / 2)^{2} \oplus \mathbb{Z} / 3$ \\
\hline-120 & 30 & $\mathbb{Z} / 2 \times \mathbb{Z} / 2$ & $\mathbb{Z}^{6}$ & $(\mathbb{Z} / 2)^{3} \oplus(\mathbb{Z} / 3)^{3}$ \\
\hline-123 & 123 & $\mathbb{Z} / 2$ & $\mathbb{Z}^{5}$ & $\mathbb{Z} / 3$ \\
\hline-127 & 127 & $\mathbb{Z} / 5$ & $\mathbb{Z}^{3}$ & $\mathbb{Z} / 2$ \\
\hline-131 & 131 & $\mathbb{Z} / 5$ & $\mathbb{Z}^{3}$ & $\mathbb{Z} / 3$ \\
\hline $\begin{array}{l}131 \\
-132\end{array}$ & $\begin{array}{r}101 \\
33\end{array}$ & $\mathbb{Z} / 2 \times \mathbb{Z} / 2$ & $\mathbb{Z}^{6}$ & $(\mathbb{Z} / 2)^{3} \oplus(\mathbb{Z} / 3)^{4}$ \\
\hline-136 & 34 & $\mathbb{Z} / 4$ & $\mathbb{Z}^{4}$ & $(\mathbb{Z} / 2)^{4} \oplus \mathbb{Z} / 3$ \\
\hline-139 & 139 & $\mathbb{Z} / 3$ & $\mathbb{Z}^{4}$ & 0 \\
\hline-143 & 143 & $\mathbb{Z} / 10$ & $\mathbb{Z}^{2}$ & $\mathbb{Z} / 2 \oplus(\mathbb{Z} / 3)^{2}$ \\
\hline-148 & 37 & $\mathbb{Z} / 2$ & $\mathbb{Z}^{6}$ & $(\mathbb{Z} / 2)^{4}$ \\
\hline-151 & 151 & $\mathbb{Z} / 7$ & $\mathbb{Z}^{3}$ & $\mathbb{Z} / 2$ \\
\hline-152 & 38 & $\mathbb{Z} / 6$ & $\mathbb{Z}^{4}$ & $\mathbb{Z} / 2 \oplus \mathbb{Z} / 3$ \\
\hline-155 & 155 & $\mathbb{Z} / 4$ & $\mathbb{Z}^{6}$ & $\mathbb{Z} / 2 \oplus \mathbb{Z} / 3$ \\
\hline-159 & 159 & $\mathbb{Z} / 10$ & $\mathbb{Z}^{4}$ & $\mathbb{Z} / 2 \oplus \mathbb{Z} / 3$ \\
\hline-163 & 163 & $\{1\}$ & $\mathbb{Z}^{6}$ & 0 \\
\hline-164 & 41 & $\mathbb{Z} / 8$ & $\mathbb{Z}^{4}$ & $(\mathbb{Z} / 2)^{2} \oplus \mathbb{Z} / 3$ \\
\hline-167 & 167 & $\mathbb{Z} / 11$ & $\mathbb{Z}^{2}$ & $\mathbb{Z} / 2 \oplus \mathbb{Z} / 3$ \\
\hline-168 & 42 & $\mathbb{Z} / 2 \times \mathbb{Z} / 2$ & $\mathbb{Z}^{9}$ & $(\mathbb{Z} / 2)^{3} \oplus(\mathbb{Z} / 3)^{2}$ \\
\hline-179 & 179 & $\mathbb{Z} / 5$ & $\mathbb{Z}^{5}$ & $\mathbb{Z} / 3$ \\
\hline-183 & 183 & $\mathbb{Z} / 8$ & $\mathbb{Z}^{6}$ & $\mathbb{Z} / 2 \oplus \mathbb{Z} / 3$ \\
\hline-184 & 46 & $\mathbb{Z} / 4$ & $\mathbb{Z}^{7}$ & $(\mathbb{Z} / 2)^{2} \oplus \mathbb{Z} / 3$ \\
\hline-187 & 187 & $\mathbb{Z} / 2$ & $\mathbb{Z}^{7}$ & $\mathbb{Z} / 3$ \\
\hline-191 & 191 & $\mathbb{Z} / 13$ & $\mathbb{Z}^{2}$ & $\mathbb{Z} / 2 \oplus \mathbb{Z} / 3$ \\
\hline
\end{tabular}


TABle 1. (Continued.)

\begin{tabular}{|c|c|c|c|c|}
\hline$\Delta$ & $m$ & Class group & $\mathrm{H}_{1}^{\text {cusp }}(\Gamma \backslash \mathcal{H} ; \mathbb{Z})$ & Farrell supplement \\
\hline-195 & 195 & $\mathbb{Z} / 2 \times \mathbb{Z} / 2$ & $\mathbb{Z}^{11}$ & $(\mathbb{Z} / 2)^{2} \oplus(\mathbb{Z} / 3)^{2}$ \\
\hline-199 & 199 & $\mathbb{Z} / 9$ & $\mathbb{Z}^{4}$ & $\mathbb{Z} / 2$ \\
\hline-203 & 203 & $\mathbb{Z} / 4$ & $\mathbb{Z}^{8}$ & $\mathbb{Z} / 2 \oplus \mathbb{Z} / 3$ \\
\hline-211 & 211 & $\mathbb{Z} / 3$ & $\mathbb{Z}^{7}$ & 0 \\
\hline-212 & 53 & $\mathbb{Z} / 6$ & $\mathbb{Z}^{8}$ & $(\mathbb{Z} / 2)^{2} \oplus \mathbb{Z} / 3$ \\
\hline-215 & 215 & $\mathbb{Z} / 14$ & $\mathbb{Z}^{4}$ & $\mathbb{Z} / 2 \oplus \mathbb{Z} / 3$ \\
\hline-219 & 219 & $\mathbb{Z} / 4$ & $\mathbb{Z}^{9}$ & $\mathbb{Z} / 2 \oplus \mathbb{Z} / 3$ \\
\hline-223 & 223 & $\mathbb{Z} / 7$ & $\mathbb{Z}^{8}$ & $(\mathbb{Z} / 2)^{3}$ \\
\hline-227 & 227 & $\mathbb{Z} / 5$ & $\mathbb{Z}^{7}$ & $\mathbb{Z} / 3$ \\
\hline-228 & 57 & $\mathbb{Z} / 2 \times \mathbb{Z} / 2$ & $\mathbb{Z}^{12}$ & $(\mathbb{Z} / 2)^{3} \oplus(\mathbb{Z} / 3)^{2}$ \\
\hline-231 & 231 & $\mathbb{Z} / 6 \times \mathbb{Z} / 2$ & $\mathbb{Z}^{9}$ & $(\mathbb{Z} / 2)^{2} \oplus(\mathbb{Z} / 3)^{2}$ \\
\hline-232 & 58 & $\mathbb{Z} / 2$ & $\mathbb{Z}^{10}$ & $(\mathbb{Z} / 2)^{2} \oplus \mathbb{Z} / 3$ \\
\hline-235 & 235 & $\mathbb{Z} / 2$ & $\mathbb{Z}^{11}$ & $(\mathbb{Z} / 2)^{3} \oplus \mathbb{Z} / 3$ \\
\hline-239 & 239 & $\mathbb{Z} / 15$ & $\mathbb{Z}^{3}$ & $\mathbb{Z} / 2 \oplus \mathbb{Z} / 3$ \\
\hline-244 & 61 & $\mathbb{Z} / 6$ & $\mathbb{Z}^{9}$ & $(\mathbb{Z} / 2)^{2}$ \\
\hline-247 & 247 & $\mathbb{Z} / 6$ & $\mathbb{Z}^{8}$ & $\mathbb{Z} / 2$ \\
\hline-248 & 62 & $\mathbb{Z} / 8$ & $\mathbb{Z}^{8}$ & $(\mathbb{Z} / 2)^{2} \oplus \mathbb{Z} / 3$ \\
\hline-251 & 251 & $\mathbb{Z} / 7$ & $\mathbb{Z}^{7}$ & $\mathbb{Z} / 3$ \\
\hline-255 & 255 & $\mathbb{Z} / 6 \times \mathbb{Z} / 2$ & $\mathbb{Z}^{11}$ & $(\mathbb{Z} / 2)^{2} \oplus(\mathbb{Z} / 3)^{3}$ \\
\hline-259 & 259 & $\mathbb{Z} / 4$ & $\mathbb{Z}^{10}$ & $\mathbb{Z} / 2 \oplus \mathbb{Z} / 3$ \\
\hline-260 & 65 & $\mathbb{Z} / 4 \times \mathbb{Z} / 2$ & $\mathbb{Z}^{12}$ & $(\mathbb{Z} / 2)^{5} \oplus(\mathbb{Z} / 3)^{2}$ \\
\hline-263 & 263 & $\mathbb{Z} / 13$ & $\mathbb{Z}^{5}$ & $\mathbb{Z} / 2 \oplus \mathbb{Z} / 3$ \\
\hline-264 & 66 & $\mathbb{Z} / 4 \times \mathbb{Z} / 2$ & $\mathbb{Z}^{12}$ & $(\mathbb{Z} / 2)^{2} \oplus(\mathbb{Z} / 3)^{3}$ \\
\hline-267 & 267 & $\mathbb{Z} / 2$ & $\mathbb{Z}^{13}$ & $\mathbb{Z} / 3$ \\
\hline-271 & 271 & $\mathbb{Z} / 11$ & $\mathbb{Z}^{6}$ & $\mathbb{Z} / 2$ \\
\hline-276 & 69 & $\mathbb{Z} / 4 \times \mathbb{Z} / 2$ & $\mathbb{Z}^{15}$ & $(\mathbb{Z} / 2)^{3} \oplus(\mathbb{Z} / 3)^{2}$ \\
\hline-280 & 70 & $\mathbb{Z} / 2 \times \mathbb{Z} / 2$ & $\mathbb{Z}^{15}$ & $(\mathbb{Z} / 2)^{3} \oplus(\mathbb{Z} / 3)^{2}$ \\
\hline-283 & 283 & $\mathbb{Z} / 3$ & $\mathbb{Z}^{10}$ & 0 \\
\hline-287 & 287 & $\mathbb{Z} / 14$ & $\mathbb{Z}^{7}$ & $(\mathbb{Z} / 2)^{2} \oplus \mathbb{Z} / 3$ \\
\hline-291 & 291 & $\mathbb{Z} / 4$ & $\mathbb{Z}^{13}$ & $\mathbb{Z} / 2 \oplus \mathbb{Z} / 3$ \\
\hline-292 & 73 & $\mathbb{Z} / 4$ & $\mathbb{Z}^{12}$ & $(\mathbb{Z} / 2)^{2} \oplus \mathbb{Z} / 3$ \\
\hline-295 & 295 & $\mathbb{Z} / 8$ & $\mathbb{Z}^{11}$ & $\mathbb{Z} / 2 \oplus \mathbb{Z} / 3$ \\
\hline-296 & 74 & $\mathbb{Z} / 10$ & $\mathbb{Z}^{9} \oplus(\mathbb{Z} / 2)^{2}$ & $(\mathbb{Z} / 2)^{2} \oplus(\mathbb{Z} / 3)^{2}$ \\
\hline-299 & 299 & $\mathbb{Z} / 8$ & $\mathbb{Z}^{10}$ & $\mathbb{Z} / 2 \oplus(\mathbb{Z} / 3)^{4}$ \\
\hline-303 & 303 & $\mathbb{Z} / 10$ & $\mathbb{Z}^{12}$ & $\mathbb{Z} / 2 \oplus \mathbb{Z} / 3$ \\
\hline-307 & 307 & $\mathbb{Z} / 3$ & $\mathbb{Z}^{11}$ & 0 \\
\hline-308 & 77 & $\mathbb{Z} / 4 \times \mathbb{Z} / 2$ & $\mathbb{Z}^{15}$ & $(\mathbb{Z} / 2)^{3} \oplus(\mathbb{Z} / 3)^{2}$ \\
\hline-311 & 311 & $\mathbb{Z} / 19$ & $\mathbb{Z}^{4}$ & $\mathbb{Z} / 2 \oplus \mathbb{Z} / 3$ \\
\hline-312 & 78 & $\mathbb{Z} / 2 \times \mathbb{Z} / 2$ & $\mathbb{Z}^{18}$ & $(\mathbb{Z} / 2)^{3} \oplus(\mathbb{Z} / 3)^{2}$ \\
\hline-319 & 319 & $\mathbb{Z} / 10$ & $\mathbb{Z}^{10}$ & $\mathbb{Z} / 2 \oplus \mathbb{Z} / 3$ \\
\hline-323 & 323 & $\mathbb{Z} / 4$ & $\mathbb{Z}^{12}$ & $\mathbb{Z} / 2 \oplus \mathbb{Z} / 3$ \\
\hline-327 & 327 & $\mathbb{Z} / 12$ & $\mathbb{Z}^{12}$ & $\mathbb{Z} / 2 \oplus \mathbb{Z} / 3$ \\
\hline-328 & 82 & $\mathbb{Z} / 4$ & $\mathbb{Z}^{13}$ & $\mathbb{Z} / 2)^{3} \oplus \mathbb{Z} / 3$ \\
\hline-331 & 331 & $\mathbb{Z} / 3$ & $\mathbb{Z}^{12}$ & $\mathbb{Z} / 3$ \\
\hline-335 & 335 & $\mathbb{Z} / 18$ & $\mathbb{Z}^{8}$ & $\mathbb{Z} / 2 \oplus \mathbb{Z} / 3$ \\
\hline-339 & 339 & $\mathbb{Z} / 6$ & $\mathbb{Z}^{15}$ & $\mathbb{Z} / 3$ \\
\hline-340 & 85 & $\mathbb{Z} / 2 \times \mathbb{Z} / 2$ & $\mathbb{Z}^{19}$ & $(\mathbb{Z} / 2)^{4} \oplus(\mathbb{Z} / 3)^{2}$ \\
\hline-344 & 86 & $\mathbb{Z} / 10$ & $\mathbb{Z}^{11} \oplus(\mathbb{Z} / 2)^{2}$ & $\mathbb{Z} / 2 \oplus \mathbb{Z} / 3$ \\
\hline-347 & 347 & $\mathbb{Z} / 5$ & $\mathbb{Z}^{12}$ & $\mathbb{Z} / 3$ \\
\hline-355 & 355 & $\mathbb{Z} / 4$ & $\mathbb{Z}^{16}$ & $\mathbb{Z} / 2 \oplus \mathbb{Z} / 3$ \\
\hline-356 & 89 & $\mathbb{Z} / 12$ & $\mathbb{Z}^{12}$ & $(\mathbb{Z} / 2)^{2} \oplus \mathbb{Z} / 3$ \\
\hline-359 & 359 & $\mathbb{Z} / 19$ & $\mathbb{Z}^{6} \oplus(\mathbb{Z} / 2)^{2}$ & $(\mathbb{Z} / 2)^{3} \oplus \mathbb{Z} / 3$ \\
\hline-367 & 367 & $\mathbb{Z} / 9$ & $\mathbb{Z}^{11} \oplus(\mathbb{Z} / 3)^{2}$ & $\mathbb{Z} / 2 \oplus \mathbb{Z} / 3$ \\
\hline-371 & 371 & $\mathbb{Z} / 8$ & $\mathbb{Z}^{14}$ & $\mathbb{Z} / 2 \oplus \mathbb{Z} / 3$ \\
\hline-372 & 93 & $\mathbb{Z} / 2 \times \mathbb{Z} / 2$ & $\mathbb{Z}^{23}$ & $(\mathbb{Z} / 2)^{3} \oplus(\mathbb{Z} / 3)^{2}$ \\
\hline-376 & 94 & $\mathbb{Z} / 8$ & $\mathbb{Z}^{14}$ & $(\mathbb{Z} / 2)^{2} \oplus \mathbb{Z} / 3$ \\
\hline-379 & 379 & $\mathbb{Z} / 3$ & $\mathbb{Z}^{14}$ & 0 \\
\hline-383 & 383 & $\mathbb{Z} / 17$ & $\mathbb{Z}^{8}$ & $\mathbb{Z} / 2 \oplus \mathbb{Z} / 3$ \\
\hline
\end{tabular}


TABLE 1. (Continued.)

\begin{tabular}{|c|c|c|c|c|}
\hline$\Delta$ & $m$ & Class group & $\mathrm{H}_{1}^{\text {cusp }}(\Gamma \backslash \mathcal{H} ; \mathbb{Z})$ & Farrell supplement \\
\hline-388 & 97 & $\mathbb{Z} / 4$ & $\mathbb{Z}^{17}$ & $(\mathbb{Z} / 2)^{2} \oplus \mathbb{Z} / 3$ \\
\hline-391 & 391 & $\mathbb{Z} / 14$ & $\mathbb{Z}^{11}$ & $(\mathbb{Z} / 2)^{2} \oplus \mathbb{Z} / 3$ \\
\hline-395 & 395 & $\mathbb{Z} / 8$ & $\mathbb{Z}^{16} \oplus(\mathbb{Z} / 2)^{2}$ & $\mathbb{Z} / 2 \oplus \mathbb{Z} / 3$ \\
\hline-399 & 399 & $\mathbb{Z} / 8 \times \mathbb{Z} / 2$ & $\mathbb{Z}^{17}$ & $(\mathbb{Z} / 2)^{4} \oplus(\mathbb{Z} / 3)^{2}$ \\
\hline-403 & 403 & $\mathbb{Z} / 2$ & $\mathbb{Z}^{17}$ & $\mathbb{Z} / 2$ \\
\hline-404 & 101 & $\mathbb{Z} / 14$ & $\mathbb{Z}^{14}$ & $(\mathbb{Z} / 2)^{4} \oplus \mathbb{Z} / 3$ \\
\hline-407 & 407 & $\mathbb{Z} / 16$ & $\mathbb{Z}^{13}$ & $\mathbb{Z} / 2 \oplus(\mathbb{Z} / 3)^{2}$ \\
\hline-408 & 102 & $\mathbb{Z} / 2 \times \mathbb{Z} / 2$ & $\mathbb{Z}^{23}$ & $(\mathbb{Z} / 2)^{2} \oplus(\mathbb{Z} / 3)^{6}$ \\
\hline-411 & 411 & $\mathbb{Z} / 6$ & $\mathbb{Z}^{19}$ & $\mathbb{Z} / 3$ \\
\hline-415 & 415 & $\mathbb{Z} / 10$ & $\mathbb{Z}^{18}$ & $\mathbb{Z} / 2 \oplus \mathbb{Z} / 3$ \\
\hline-419 & 419 & $\mathbb{Z} / 9$ & $\mathbb{Z}^{13}$ & $(\mathbb{Z} / 3)^{3}$ \\
\hline-420 & 105 & $\mathbb{Z} / 2 \times \mathbb{Z} / 2 \times \mathbb{Z} / 2$ & $\mathbb{Z}^{33}$ & $(\mathbb{Z} / 2)^{8} \oplus(\mathbb{Z} / 3)^{4}$ \\
\hline-424 & 106 & $\mathbb{Z} / 6$ & $\mathbb{Z}^{17} \oplus(\mathbb{Z} / 2)^{2}$ & $(\mathbb{Z} / 2)^{2} \oplus \mathbb{Z} / 3$ \\
\hline-427 & 427 & $\mathbb{Z} / 2$ & $\mathbb{Z}^{19}$ & $(\mathbb{Z} / 2)^{3}$ \\
\hline-431 & 431 & $\mathbb{Z} / 21$ & $\mathbb{Z}^{8} \oplus \mathbb{Z} / 2$ & $\mathbb{Z} / 2 \oplus \mathbb{Z} / 3$ \\
\hline-435 & 435 & $\mathbb{Z} / 2 \times \mathbb{Z} / 2$ & $\mathbb{Z}^{27}$ & $(\mathbb{Z} / 2)^{2} \oplus(\mathbb{Z} / 3)^{6}$ \\
\hline-436 & 109 & $\mathbb{Z} / 6$ & $\mathbb{Z}^{19} \oplus(\mathbb{Z} / 2)^{2}$ & $(\mathbb{Z} / 2)^{2}$ \\
\hline-439 & 439 & $\mathbb{Z} / 15$ & $\mathbb{Z}^{11}$ & $(\mathbb{Z} / 2)^{5}$ \\
\hline-440 & 110 & $\mathbb{Z} / 6 \times \mathbb{Z} / 2$ & $\mathbb{Z}^{20}$ & $(\mathbb{Z} / 2)^{3} \oplus(\mathbb{Z} / 3)^{2}$ \\
\hline-443 & 443 & $\mathbb{Z} / 5$ & $\mathbb{Z}^{16}$ & $\mathbb{Z} / 2 \oplus \mathbb{Z} / 3$ \\
\hline-447 & 447 & $\mathbb{Z} / 14$ & $\mathbb{Z}^{18}$ & $\mathbb{Z} / 2 \oplus \mathbb{Z} / 3$ \\
\hline-451 & 451 & $\mathbb{Z} / 6$ & $\mathbb{Z}^{17}$ & $\mathbb{Z} / 3$ \\
\hline-452 & 113 & $\mathbb{Z} / 8$ & $\mathbb{Z}^{19} \oplus(\mathbb{Z} / 2)^{2}$ & $(\mathbb{Z} / 2)^{2} \oplus \mathbb{Z} / 3$ \\
\hline-455 & 455 & $\mathbb{Z} / 10 \times \mathbb{Z} / 2$ & $\mathbb{Z}^{19} \oplus(\mathbb{Z} / 2)^{2}$ & $(\mathbb{Z} / 2)^{2} \oplus(\mathbb{Z} / 3)^{2}$ \\
\hline-456 & 114 & $\mathbb{Z} / 4 \times \mathbb{Z} / 2$ & $\mathbb{Z}^{24} \oplus(\mathbb{Z} / 2)^{2}$ & $(\mathbb{Z} / 2)^{2} \oplus(\mathbb{Z} / 3)^{4}$ \\
\hline-463 & 463 & $\mathbb{Z} / 7$ & $\mathbb{Z}^{16}$ & $\mathbb{Z} / 2$ \\
\hline-467 & 467 & $\mathbb{Z} / 7$ & $\mathbb{Z}^{16}$ & $\mathbb{Z} / 3$ \\
\hline-471 & 471 & $\mathbb{Z} / 16$ & $\mathbb{Z}^{18}$ & $\mathbb{Z} / 2 \oplus \mathbb{Z} / 3$ \\
\hline-472 & 118 & $\mathbb{Z} / 6$ & $\mathbb{Z}^{19} \oplus(\mathbb{Z} / 2)^{2}$ & $\mathbb{Z} / 2 \oplus \mathbb{Z} / 3$ \\
\hline-479 & 479 & $\mathbb{Z} / 25$ & $\mathbb{Z}^{8} \oplus(\mathbb{Z} / 3)^{2}$ & $\mathbb{Z} / 2 \oplus \mathbb{Z} / 3$ \\
\hline-483 & 483 & $\mathbb{Z} / 2 \times \mathbb{Z} / 2$ & $\mathbb{Z}^{29}$ & $(\mathbb{Z} / 2)^{2} \oplus(\mathbb{Z} / 3)^{2}$ \\
\hline-487 & 487 & $\mathbb{Z} / 7$ & $\mathbb{Z}^{17} \oplus(\mathbb{Z} / 13)^{2}$ & $\mathbb{Z} / 2$ \\
\hline-488 & 122 & $\mathbb{Z} / 10$ & $\mathbb{Z}^{18} \oplus(\mathbb{Z} / 2)^{2}$ & $(\mathbb{Z} / 2)^{2} \oplus(\mathbb{Z} / 3)^{2}$ \\
\hline-491 & 491 & $\mathbb{Z} / 9$ & $\mathbb{Z}^{16}$ & $\mathbb{Z} / 3$ \\
\hline-499 & 499 & $\mathbb{Z} / 3$ & $\mathbb{Z}^{19} \oplus(\mathbb{Z} / 3)^{2}$ & $(\mathbb{Z} / 2)^{2}$ \\
\hline
\end{tabular}

Definition 1. A pair of elements $(\mu, \lambda) \in \mathcal{O}^{2}$ is called unimodular if the ideal sum $\mu \mathcal{O}+\lambda \mathcal{O}$ equals $\mathcal{O}$.

The boundary of $\mathcal{H}$ is the Riemann sphere $\partial \mathcal{H}=\mathbb{C} \cup\{\infty\}$ (as a set), which contains the complex plane $\mathbb{C}$. The totally geodesic surfaces in $\mathcal{H}$ are the Euclidean vertical planes (we define vertical as orthogonal to the complex plane) and the Euclidean hemispheres centred on the complex plane.

Notation 2. Given a unimodular pair $(\mu, \lambda) \in \mathcal{O}^{2}$ with $\mu \neq 0$, let $S_{\mu, \lambda} \subset \mathcal{H}$ denote the hemisphere given by the equation $|\mu z-\lambda|^{2}+|\mu|^{2} \zeta^{2}=1$.

This hemisphere has centre $\lambda / \mu$ on the complex plane $\mathbb{C}$ and radius $1 /|\mu|$.

Let

$$
\begin{aligned}
B:=\left\{(z, \zeta) \in \mathcal{H}: \text { The inequality }|\mu z-\lambda|^{2}+|\mu|^{2} \zeta^{2} \geqslant 1\right. \\
\left.\quad \text { is fulfilled for all unimodular pairs }(\mu, \lambda) \in \mathcal{O}^{2} \text { with } \mu \neq 0\right\} .
\end{aligned}
$$

Then $B$ is the set of points in $\mathcal{H}$ which lie above or on all hemispheres $S_{\mu, \lambda}$.

Lemma 3 (Swan [32]). The set $B$ contains representatives for all of the orbits of points under the action of $\mathrm{SL}_{2}(\mathcal{O})$ on $\mathcal{H}$. 
The action extends continuously to the boundary $\partial \mathcal{H}$, which is a Riemann sphere.

In $\Gamma:=\mathrm{SL}_{2}\left(\mathcal{O}_{-m}\right)$, consider the stabilizer subgroup $\Gamma_{\infty}$ of the point $\infty \in \partial \mathcal{H}$. In the cases $m=1$ and $m=3$, the latter group contains some rotation matrices such as $\left(\begin{array}{cc}0 & \sqrt{-1} \\ \sqrt{-1} & 0\end{array}\right)$, which we want to exclude. These two cases have been treated in $[\mathbf{1 5}, \mathbf{2 7}]$ among others, and we assume $m \neq 1, m \neq 3$ throughout the remainder of this article. Then,

$$
\Gamma_{\infty}=\left\{ \pm\left(\begin{array}{ll}
1 & \lambda \\
0 & 1
\end{array}\right) \mid \lambda \in \mathcal{O}\right\}
$$

which performs translations by the elements of $\mathcal{O}$ with respect to the Euclidean geometry of the upper-half space $\mathcal{H}$.

Notation 4. A fundamental domain for $\Gamma_{\infty}$ in the complex plane (as a subset of $\partial \mathcal{H}$ ) is given by the rectangle

$$
D_{0}:= \begin{cases}\{x+y \sqrt{-m} \in \mathbb{C} \mid 0 \leqslant x \leqslant 1,0 \leqslant y \leqslant 1\}, & m \equiv 1 \text { or } 2 \bmod 4, \\ \left\{x+y \sqrt{-m} \in \mathbb{C} \mid \frac{-1}{2} \leqslant x \leqslant \frac{1}{2}, 0 \leqslant y \leqslant \frac{1}{2}\right\}, & m \equiv 3 \bmod 4 .\end{cases}
$$

TABLE 2. The cuspidal homology $\mathrm{H}_{1}^{\text {cusp }}(\Gamma \backslash \mathcal{H} ; \mathbb{Z})$, with its torsion decomposed into prime power

\begin{tabular}{|c|c|c|c|c|}
\hline$\Delta$ & $m$ & Class group & $\mathrm{H}_{1}^{\text {cusp }}(\Gamma \backslash \mathcal{H} ; \mathbb{Z})$ & Farrell supplement \\
\hline-520 & 130 & $\mathbb{Z} / 2 \times \mathbb{Z} / 2$ & $\mathbb{Z}^{28} \oplus(\mathbb{Z} / 2)^{2}$ & $(\mathbb{Z} / 2)^{4} \oplus(\mathbb{Z} / 3)^{2}$ \\
\hline-523 & 523 & $\mathbb{Z} / 5$ & $\mathbb{Z}^{19}$ & 0 \\
\hline-532 & 133 & $\mathbb{Z} / 2 \times \mathbb{Z} / 2$ & $\mathbb{Z}^{29}$ & $(\mathbb{Z} / 2)^{3} \oplus(\mathbb{Z} / 3)^{2}$ \\
\hline-547 & 547 & $\mathbb{Z} / 3$ & $\mathbb{Z}^{21} \oplus(\mathbb{Z} / 2)^{2}$ & $(\mathbb{Z} / 3)^{2}$ \\
\hline-555 & 555 & $\mathbb{Z} / 2 \times \mathbb{Z} / 2$ & $\mathbb{Z}^{35}$ & $(\mathbb{Z} / 2)^{2} \oplus(\mathbb{Z} / 3)^{2}$ \\
\hline-568 & 142 & $\mathbb{Z} / 4$ & $\mathbb{Z}^{25} \oplus(\mathbb{Z} / 2)^{2}$ & $(\mathbb{Z} / 2)^{6} \oplus \mathbb{Z} / 3$ \\
\hline-571 & 571 & $\mathbb{Z} / 5$ & $\mathbb{Z}^{23}$ & 0 \\
\hline-595 & 595 & $\mathbb{Z} / 2 \times \mathbb{Z} / 2$ & $\mathbb{Z}^{33}$ & $(\mathbb{Z} / 2)^{2} \oplus(\mathbb{Z} / 3)^{4}$ \\
\hline-619 & 619 & $\mathbb{Z} / 5$ & $\mathbb{Z}^{23} \oplus(\mathbb{Z} / 3)^{2}$ & 0 \\
\hline-627 & 627 & $\mathbb{Z} / 2 \times \mathbb{Z} / 2$ & $\mathbb{Z}^{35}$ & $(\mathbb{Z} / 3)^{2}$ \\
\hline-643 & 643 & $\mathbb{Z} / 3$ & $\mathbb{Z}^{27}$ & $\mathbb{Z} / 3$ \\
\hline-667 & 667 & $\mathbb{Z} / 4$ & $\mathbb{Z}^{28}$ & $\mathbb{Z} / 2 \oplus \mathbb{Z} / 3$ \\
\hline-683 & 683 & $\mathbb{Z} / 5$ & $\mathbb{Z}^{26}$ & $\mathbb{Z} / 3$ \\
\hline-691 & 691 & $\mathbb{Z} / 5$ & $\mathbb{Z}^{26} \oplus(\mathbb{Z} / 7)^{2}$ & 0 \\
\hline-696 & 174 & $\mathbb{Z} / 6 \times \mathbb{Z} / 2$ & $\mathbb{Z}^{38}$ & $(\mathbb{Z} / 2)^{3} \oplus(\mathbb{Z} / 3)^{3}$ \\
\hline-715 & 715 & $\mathbb{Z} / 2 \times \mathbb{Z} / 2$ & $\mathbb{Z}^{39}$ & $(\mathbb{Z} / 2)^{2} \oplus(\mathbb{Z} / 3)^{2}$ \\
\hline-723 & 723 & $\mathbb{Z} / 4$ & $\mathbb{Z}^{37} \oplus(\mathbb{Z} / 2)^{2}$ & $\mathbb{Z} / 2 \oplus \mathbb{Z} / 3$ \\
\hline-739 & 739 & $\mathbb{Z} / 5$ & $\mathbb{Z}^{28}$ & 0 \\
\hline-760 & 190 & $\mathbb{Z} / 2 \times \mathbb{Z} / 2$ & $\mathbb{Z}^{42} \oplus(\mathbb{Z} / 2)^{2}$ & $(\mathbb{Z} / 2)^{3} \oplus(\mathbb{Z} / 3)^{2}$ \\
\hline-763 & 763 & $\mathbb{Z} / 4$ & $\mathbb{Z}^{34}$ & $\mathbb{Z} / 2 \oplus \mathbb{Z} / 3$ \\
\hline-787 & 787 & $\mathbb{Z} / 5$ & $\mathbb{Z}^{30}$ & 0 \\
\hline-795 & 795 & $\mathbb{Z} / 2 \times \mathbb{Z} / 2$ & $\mathbb{Z}^{51}$ & $(\mathbb{Z} / 2)^{2} \oplus(\mathbb{Z} / 3)^{3}$ \\
\hline-883 & 883 & $\mathbb{Z} / 3$ & $\mathbb{Z}^{35}$ & 0 \\
\hline-907 & 907 & $\mathbb{Z} / 3$ & $\mathbb{Z}^{36} \oplus(\mathbb{Z} / 13)^{2}$ & 0 \\
\hline-947 & 947 & $\mathbb{Z} / 5$ & $\mathbb{Z}^{37} \oplus(\mathbb{Z} / 89)^{2}$ & $\mathbb{Z} / 3$ \\
\hline-955 & 955 & $\mathbb{Z} / 4$ & $\mathbb{Z}^{46} \oplus(\mathbb{Z} / 2)^{4} \oplus(\mathbb{Z} / 3)^{2}$ & $\mathbb{Z} / 2 \oplus \mathbb{Z} / 3$ \\
\hline-1003 & 1003 & $\mathbb{Z} / 4$ & $\mathbb{Z}^{44} \oplus(\mathbb{Z} / 3)^{2}$ & $\mathbb{Z} / 2 \oplus \mathbb{Z} / 3$ \\
\hline-1027 & 1027 & $\mathbb{Z} / 4$ & $\mathbb{Z}^{44} \oplus(\mathbb{Z} / 2)^{2}$ & $\mathbb{Z} / 2 \oplus(\mathbb{Z} / 3)^{3}$ \\
\hline-1051 & 1051 & $\mathbb{Z} / 5$ & $\mathbb{Z}^{43} \oplus(\mathbb{Z} / 13)^{2}$ & 0 \\
\hline-1123 & 1123 & $\mathbb{Z} / 5$ & $\mathbb{Z}^{44} \oplus(\mathbb{Z} / 7)^{2}$ & 0 \\
\hline-1227 & 1227 & $\mathbb{Z} / 4$ & $\mathbb{Z}^{65} \oplus\left(\mathbb{Z} / 2^{2}\right)^{2}$ & $\mathbb{Z} / 2 \oplus \mathbb{Z} / 3$ \\
\hline-1243 & 1243 & $\mathbb{Z} / 4$ & $\mathbb{Z}^{54} \oplus(\mathbb{Z} / 3)^{4}$ & $\mathbb{Z} / 2 \oplus \mathbb{Z} / 3$ \\
\hline-1387 & 1387 & $\mathbb{Z} / 4$ & $\mathbb{Z}^{58} \oplus(\mathbb{Z} / 167)^{2}$ & $\mathbb{Z} / 2 \oplus(\mathbb{Z} / 3)^{3}$ \\
\hline-1411 & 1411 & $\mathbb{Z} / 4$ & $\mathbb{Z}^{60} \oplus\left(\mathbb{Z} / 2^{4}\right)^{2} \oplus(\mathbb{Z} / 43)^{2}$ & $\mathbb{Z} / 2 \oplus \mathbb{Z} / 3$ \\
\hline-1507 & 1507 & $\mathbb{Z} / 4$ & $\mathbb{Z}^{66} \oplus(\mathbb{Z} / 3)^{2} \oplus(\mathbb{Z} / 5)^{4}$ & $\mathbb{Z} / 2 \oplus \mathbb{Z} / 3$ \\
\hline-1555 & 1555 & $\mathbb{Z} / 4$ & $\mathbb{Z}^{76} \oplus\left(\mathbb{Z} / 2^{2}\right)^{8} \oplus(\mathbb{Z} / 11)^{2}$ & $\mathbb{Z} / 2 \oplus \mathbb{Z} / 3$ \\
\hline-1723 & 1723 & $\mathbb{Z} / 5$ & $\mathbb{Z}^{69} \oplus(\mathbb{Z} / 7)^{2} \oplus(\mathbb{Z} / 23)^{2} \oplus(\mathbb{Z} / 883)^{2}$ & 0 \\
\hline-1747 & 1747 & $\mathbb{Z} / 5$ & $\mathbb{Z}^{70} \oplus(\mathbb{Z} / 80737)^{2}$ & $(\mathbb{Z} / 3)^{2}$ \\
\hline-1867 & 1867 & $\mathbb{Z} / 5$ & $\mathbb{Z}^{75} \oplus(\mathbb{Z} / 2)^{4} \oplus\left(\mathbb{Z} / 7^{2}\right)^{2} \oplus(\mathbb{Z} / 137)^{2}$ & 0 \\
\hline
\end{tabular}
factors, for some greater absolute values of the discriminant $\Delta$. 
A fundamental domain for $\Gamma_{\infty}$ in $\mathcal{H}$ is given by

$$
D_{\infty}:=\left\{(z, \zeta) \in \mathcal{H} \mid z \in D_{0}\right\}
$$

Definition 5. We define the Bianchi fundamental polyhedron as

$$
D:=D_{\infty} \cap B .
$$

It is a polyhedron in hyperbolic space up to the missing vertex $\infty$, and up to missing vertices at the singular points if $\mathcal{O}$ is not a principal ideal domain (see $\S 6.2$ ). As Lemma 3 states $\Gamma \cdot B=\mathcal{H}$, and as $\Gamma_{\infty} \cdot D_{\infty}=\mathcal{H}$ yields $\Gamma_{\infty} \cdot D=B$, we have $\Gamma \cdot D=\mathcal{H}$. We observe the following notion of strictness of the fundamental domain: the interior of the Bianchi fundamental polyhedron contains no two points which are identified by $\Gamma$. Swan proves the following theorem, which implies that the boundary of the Bianchi fundamental polyhedron consists of finitely many cells.

Theorem 6 (Swan [32]). There is only a finite number of unimodular pairs $(\lambda, \mu)$ such that the intersection of $S_{\mu, \lambda}$ with the Bianchi fundamental polyhedron is non-empty.

Swan further proves a corollary, from which it can be deduced that the action of $\Gamma$ on $\mathcal{H}$ is properly discontinuous.

\section{The Flöge cellular complex}

In order to obtain a cell complex with compact quotient space, we proceed in the following way due to Flöge [11]. The boundary of $\mathcal{H}$ is the Riemann sphere $\partial \mathcal{H}$, which, as a topological space, is made up of the complex plane $\mathbb{C}$ compactified with the cusp $\infty$. The totally geodesic surfaces in $\mathcal{H}$ are the Euclidean vertical planes (we define vertical as orthogonal to the complex plane) and the Euclidean hemispheres centred on the complex plane. The action of the Bianchi groups extends continuously to the boundary $\partial \mathcal{H}$. Consider the cellular structure on $\mathcal{H}$ induced by the $\Gamma$-images of the Bianchi fundamental polyhedron. The cellular closure of this cell complex in $\mathcal{H} \cup \partial \mathcal{H}$ consists of $\mathcal{H}$ and $(\mathbb{Q}(\sqrt{-m}) \cup\{\infty\}) \subset(\mathbb{C} \cup\{\infty\}) \cong \partial \mathcal{H}$. The $\mathrm{SL}_{2}\left(\mathcal{O}_{-m}\right)$-orbit of a cusp $\lambda / \mu$ in $(\mathbb{Q}(\sqrt{-m}) \cup\{\infty\})$ corresponds to the ideal class $[(\lambda, \mu)]$ of $\mathcal{O}_{-m}$. It is well-known that this does not depend on the choice of the representative $\lambda / \mu$. We extend our cell complex to a cell complex $\widetilde{X}$ by joining to it, in the case that $\mathcal{O}_{-m}$ is not a principal ideal domain, the $\mathrm{SL}_{2}\left(\mathcal{O}_{-m}\right)$-orbits of the cusps $\lambda / \mu$ for which the ideal $(\lambda, \mu)$ is not principal. At these cusps, we equip $\widetilde{X}$ with the 'horoball topology' described in [11]. This simply means that the set of cusps, which is discrete in $\partial \mathcal{H}$, is located at the hyperbolic extremities of $\widetilde{X}$ : no neighbourhood of a cusp, except the whole $\widetilde{X}$, contains any other cusp.

We retract $\widetilde{X}$ in the following $\mathrm{SL}_{2}\left(\mathcal{O}_{-m}\right)$-equivariant way. On the Bianchi fundamental polyhedron, the retraction is given by the vertical projection (away from the cusp $\infty$ ) onto its facets which are closed in $\mathcal{H} \cup \partial \mathcal{H}$. The latter are the facets which do not touch the cusp $\infty$. They are the bottom facets with respect to our vertical direction. The retraction is continued on $\mathcal{H}$ by the group action. It is proven in [10] that this retraction is continuous. We denote by $X$ the retract of $\widetilde{X}$.

Notation 7 . We will call this 2-dimensional retract $X$ the retracted Flöge cellular complex.

Note that in the principal ideal domain cases, $X$ is a retract of the original cellular structure on $\mathcal{H}$, obtained by contracting the Bianchi fundamental polyhedron onto its cells which do not touch the boundary of $\mathcal{H}$. Hence $X$ coincides with Mendoza's complex [15] in those cases. In [23], it is checked that the retracted Flöge cellular complex is contractible. 


\section{Connecting Flöge cell complex and Borel-Serre compactification}

Let $\Gamma$ be a Bianchi group with $\mathcal{O}$ admitting as only units $\{ \pm 1\}$, i.e. we suppose that $\mathcal{O}$ is not the Gaussian or Eisenstein integers. In the latter two cases, the problem of the singular cusps treated here does not occur in any case. We make use of the Borel-Serre compactification [31] for $\Gamma \backslash \mathcal{H}$. Recall that in this case, the Borel-Serre compactification joins a 2-torus $\mathbb{T}$ to $\Gamma \backslash \mathcal{H}$ at every cusp. Details are given in $[\mathbf{2 2}]$. Let $\mathbb{T}_{i}$ be the torus attached at the cusp $i$ of $\Gamma$. We decompose $\mathbb{T}_{i}$ in the classical way into a 2-cell, two 1-cells and a vertex. Let $x_{i}$ and $y_{i}$ denote the cycles generating $\mathrm{H}_{1}\left(\mathbb{T}_{i}\right)$; they are given by the two 1-cells. Let $P$ be the Bianchi fundamental polyhedron of $\Gamma$. Write 'hyp. cells' for cells in the interior of hyperbolic space. Denote by $\widetilde{\partial}$ the boundary operator for the cell complex $\widetilde{X}$. Consider following the short exact sequence of chain complexes that we obtain from collapsing the singular tori.

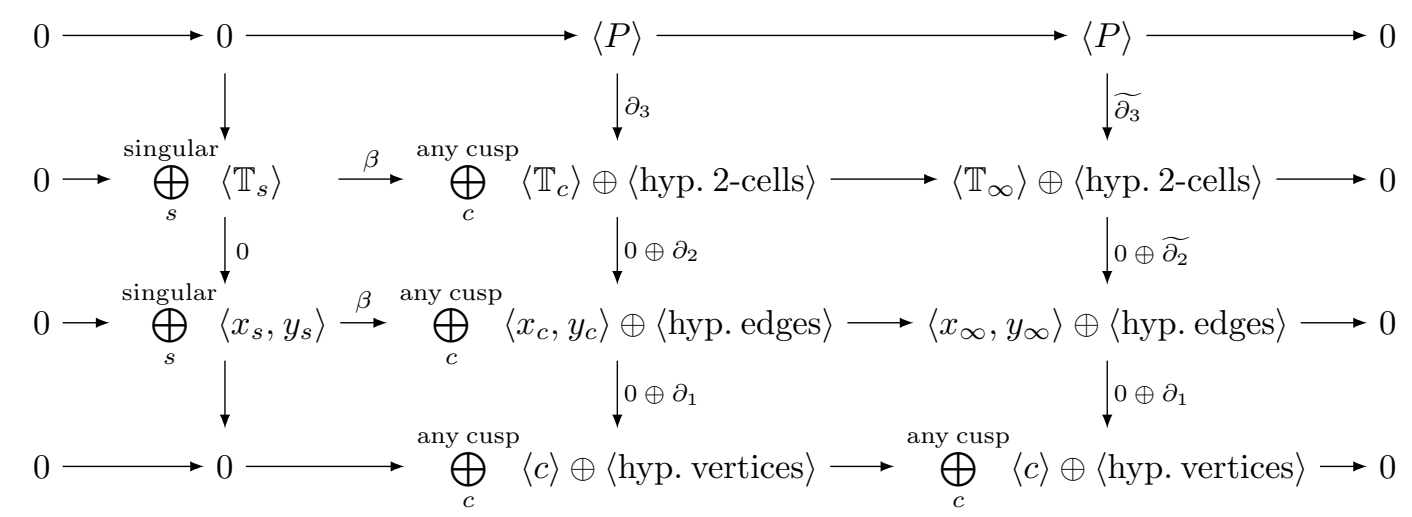

Poincaré's theorem on fundamental polyhedra tells us that $\partial_{3}(P)=\left\langle\bigcup_{c}^{\text {any cusp }} \mathbb{T}_{c}\right\rangle$ and, hence, $\widetilde{\partial}_{3}(P)=\left\langle\mathbb{T}_{\infty}\right\rangle$. From $[\mathbf{2 2}]$, we see that for every cusp $c$, there is a chain of hyperbolic 2-cells that we denote by $\operatorname{ch}\left(x_{c}\right)$ and which is mapped to the cycle $x_{c}$ by $\partial_{2}$. Furthermore, $y_{c}$ is in the cokernel of $\partial_{2}$ (of course, this holds up to the appropriate permutation of the labels $x_{c}$ and $\left.y_{c}\right)$. This implies that $\widetilde{\partial}_{2}\left(\operatorname{ch}\left(x_{\infty}\right)\right)=x_{\infty}$ and $y_{\infty}$ is in the cokernel of $\widetilde{\partial}_{2}$. As the quotient space is path-wise connected, the cokernel of $\partial_{1}$ is isomorphic to $\mathbb{Z}$. The above information tells us that the long exact sequence induced on integral homology by the map $\beta$ concentrates in the following diagram.

$$
\begin{aligned}
0 \longrightarrow \bigoplus_{s}\left\langle\mathbb{T}_{s}\right\rangle \stackrel{\beta_{2}}{\longrightarrow}\left(\bigoplus_{c}^{\text {any cusp }}\left\langle\mathbb{T}_{c}\right\rangle\right) /\left\langle\cup_{c} \mathbb{T}_{c}\right\rangle \oplus \mathrm{H}_{2}^{\text {cusp }} \longrightarrow \mathrm{H}_{2}^{\text {cusp }} \bigoplus_{s}\left\langle\operatorname{ch}\left(x_{s}\right)\right\rangle \longrightarrow \\
\longrightarrow \bigoplus_{s}\left\langle x_{s}, y_{s}\right\rangle \longrightarrow \mathrm{\beta}_{1} \longrightarrow \bigoplus_{c}\left\langle y_{c}\right\rangle \oplus \mathrm{H}_{1}^{\text {cusp }} \oplus\left\langle y_{\infty}\right\rangle \longrightarrow
\end{aligned}
$$

Here the maps without labels are the obvious restriction maps making the sequence exact and $\mathrm{H}_{1}^{\text {cusp }}$ and $\mathrm{H}_{2}^{\text {cusp }}$ are generated by cycles from the interior of $\Gamma \backslash \mathcal{H}$.

Note that $\mathrm{H}_{2}^{\text {cusp }} \bigoplus_{s}\left\langle\operatorname{ch}\left(x_{s}\right)\right\rangle$ is non-naturally isomorphic to

$$
\left.\left(\bigoplus_{c}^{\text {any cusp }}\left\langle\mathbb{T}_{c}\right\rangle\right)\right|_{\left\langle\cup_{c} \mathbb{T}_{c}\right\rangle} \oplus \mathrm{H}_{2}^{\text {cusp }},
$$

namely collapsing a torus $\mathbb{T}_{s}$ moves its 2-cycle into a bubble $\operatorname{ch}\left(x_{s}\right)$ emerging adjacent to the singular cusp $s$ in the Flöge complex. 


\section{The equivariant spectral sequence to group homology}

Let $\Gamma:=\mathrm{PSL}_{2}\left(\mathcal{O}_{-m}\right)$, and let $X$ be the retracted Flöge cellular complex of $\S 3$, the cell structure of which we subdivide until the cells are fixed pointwise by their stabilizers. We describe now how to assemble the homology of the Borel-Serre compactified quotient space (see the previous section) and the Farrell cohomology of $\Gamma$, for which general formulae have been given in $[\mathbf{2 1}]$ (based on $[\mathbf{2 0}]$ ), to the full group homology of $\Gamma$ with trivial $\mathbb{Z}$-coefficients. We proceed by following $[\mathbf{4}, \mathrm{VII}]$ and $[\mathbf{2 7}]$. Let us consider the homology $\mathrm{H}_{*}(\Gamma ; C \bullet(X))$ of $\Gamma$ with coefficients in the cellular chain complex $C \bullet(X)$ associated to $X$; and call it the $\Gamma$-equivariant homology of $X$. As $X$ is contractible, the map $X \rightarrow$ pt. to the point pt. induces an isomorphism

$$
\mathrm{H}_{*}\left(\Gamma ; C_{\bullet}(X)\right) \rightarrow \mathrm{H}_{*}\left(\Gamma ; C_{\bullet}(\text { pt. })\right) \cong \mathrm{H}_{*}(\Gamma ; \mathbb{Z}) .
$$

Denote by $X^{p}$ the set of $p$-cells of $X$, and make use of that the stabilizer $\Gamma_{\sigma}$ in $\Gamma$ of any $p$-cell $\sigma$ of $X$ fixes $\sigma$ pointwise. Then from

$$
C_{p}(X)=\bigoplus_{\sigma \in X^{p}} \mathbb{Z} \cong \bigoplus_{\sigma \in \Gamma \backslash X^{p}} \operatorname{Ind}_{\Gamma_{\sigma}}^{\Gamma} \mathbb{Z}
$$

Shapiro's lemma yields

$$
\mathrm{H}_{q}\left(\Gamma ; C_{p}(X)\right) \cong \bigoplus_{\sigma \in \Gamma \backslash X^{p}} \mathrm{H}_{q}\left(\Gamma_{\sigma} ; \mathbb{Z}\right)
$$

and the equivariant Leray/Serre spectral sequence takes the form

$$
E_{p, q}^{1}=\bigoplus_{\sigma \in \Gamma \backslash X^{p}} \mathrm{H}_{q}\left(\Gamma_{\sigma} ; \mathbb{Z}\right) \Longrightarrow \mathrm{H}_{p+q}\left(\Gamma ; C_{\bullet}(X)\right),
$$

converging to the $\Gamma$-equivariant homology of $X$, which is, as we have already seen, isomorphic to $\mathrm{H}_{p+q}(\Gamma ; \mathbb{Z})$ with the trivial action on the coefficients $\mathbb{Z}$.

As in degrees above the virtual cohomological dimension, which is two for the Bianchi groups, the group homology is isomorphic to the Farrell cohomology, we obtain the isomorphism type from the above-mentioned general formulae.

In the lower degrees $q \in\{0,1,2\}$, the following terms remain on the $E^{2}$-page, which is concentrated in the columns $p=0,1,2$ :

$$
\begin{aligned}
& q=2 \quad \bigoplus_{s \text { singular }} \mathbb{Z} \oplus 2 \text {-torsion } \oplus 3 \text {-torsion } \quad \text { 2-torsion } \oplus 3 \text {-torsion } \quad 0 \\
& \begin{array}{ccc}
q=1 \quad \bigoplus_{s \text { singular }} \mathbb{Z}^{2} \oplus \text { Farrell supplement } & \begin{array}{c}
\text { 2-torsion } \oplus \text { 3-torsion } \\
d_{2,0}^{2}
\end{array} \\
q=0 & \mathrm{H}_{1}(\Gamma \backslash X ; \mathbb{Z}) & \mathrm{H}_{2}(\Gamma \backslash X ; \mathbb{Z})
\end{array}
\end{aligned}
$$

where the 'Farrell supplement' is the cokernel of the map

$$
\bigoplus_{\sigma \in \Gamma \backslash X^{0}} \mathrm{H}_{1}\left(\Gamma_{\sigma} ; \mathbb{Z}\right) \stackrel{d_{1,1}^{1}}{\bigoplus_{\sigma \in \Gamma \backslash X^{1}}} \mathrm{H}_{1}\left(\Gamma_{\sigma} ; \mathbb{Z}\right)
$$

induced by inclusion of finite cell stabilizers. As the cells are fixed pointwise by their stabilizers, we see that for $q>0$, the $E_{p, q}^{1}$-terms are concentrated in the two columns $p=0$ and $p=1$. We compute the bottom row $(q=0)$ of the above spectral sequence as the homology of the quotient space $\Gamma \backslash X$. Then we infer from $\S 4$ that the rational rank of the differential $d_{2,0}^{2}$ is the number of non-trivial ideal classes of $\mathcal{O}_{-m}$. 
Let us use Serre's decomposition of the homology group $H_{1}(\Gamma \backslash \mathcal{H} ; \mathbb{Z})$ into the direct sum of $\mathrm{H}_{1}^{\text {cusp }}(\Gamma \backslash \mathcal{H} ; \mathbb{Z})$ and the free Abelian group with one generator for each element of the class group of $\mathcal{O}_{-m}$. Then using the long exact sequence of $\S 4$, we see that $\mathrm{H}_{1}(\Gamma \backslash X ; \mathbb{Z}) \cong$ $\mathrm{H}_{1}^{\text {cusp }}(\Gamma \backslash \mathcal{H} ; \mathbb{Z}) \oplus \mathbb{Z}$. This has made it possible to compute $\mathrm{H}_{1}^{\text {cusp }}(\Gamma \backslash \mathcal{H} ; \mathbb{Z})$ from the quotient space of the retracted Flöge cellular complex in Tables 1 and 2. Finally, the group homology $\mathrm{H}_{1}(\Gamma ; \mathbb{Z})$ is an extension of $\mathrm{H}_{1}(\Gamma \backslash \mathcal{H} ; \mathbb{Z})$ by a quotient of the Farrell supplement.

\section{Swan's concept to determine the Bianchi fundamental polyhedron}

This section recalls Richard G. Swan's work [32], which gives a concept, from the theoretical viewpoint, for an algorithm to compute the Bianchi fundamental polyhedron. The set $B$ which determines the Bianchi fundamental polyhedron has been defined using infinitely many hemispheres. But we will see that only a finite number of them are significant for this purpose and need to be computed. We will state a criterion for what is an appropriate choice that gives us precisely the set $B$. This criterion is easy to verify in practice. Suppose that we have made a finite selection of $n$ hemispheres. The index $i$ running from 1 through $n$, we denote the $i$ th hemisphere by $S\left(\alpha_{i}\right)$, where $\alpha_{i}$ is its centre and given by a fraction $\alpha_{i}=\lambda_{i} / \mu_{i}$ in the number field $\mathbb{Q}(\sqrt{-m})$. Here, we require the ideal $\left(\lambda_{i}, \mu_{i}\right)$ to be the whole ring of integers $\mathcal{O}$. This requirement is just the one already made for all of the hemispheres in the definition of $B$. Now, we can do an approximation of Notation 2 , using, modulo the translation group $\Gamma_{\infty}$, a finite number of hemispheres.

Notation 8. Let $B\left(\alpha_{1}, \ldots, \alpha_{n}\right):=\left\{(z, \zeta) \in \mathcal{H}\right.$ : the inequality $|\mu z-\lambda|^{2}+|\mu|^{2} \zeta^{2} \geqslant 1$ is fulfilled for all unimodular pairs $(\mu, \lambda) \in \mathcal{O}^{2}$ with $\lambda / \mu=\alpha_{i}+\gamma$, for some $i \in\{1, \ldots, n\}$ and some $\gamma \in \mathcal{O}\}$. Then $B\left(\alpha_{1}, \ldots, \alpha_{n}\right)$ is the set of all points in $\mathcal{H}$ lying above or on all hemispheres $S\left(\alpha_{i}+\gamma\right), i=1, \ldots, n$; for any $\gamma \in \mathcal{O}$.

The intersection $B\left(\alpha_{1}, \ldots, \alpha_{n}\right) \cap D_{\infty}$ with the fundamental domain $D_{\infty}$ for the translation group $\Gamma_{\infty}$, is our candidate to equal the Bianchi fundamental polyhedron.

\subsection{Convergence of the approximation}

We will give a method to decide when $B\left(\alpha_{1}, \ldots, \alpha_{n}\right)=B$. This gives us an effective way to find $B$ by adding more and more elements to the set $\left\{\alpha_{1}, \ldots, \alpha_{n}\right\}$ until we find $B\left(\alpha_{1}, \ldots, \alpha_{n}\right)=B$. We consider the boundary $\partial B\left(\alpha_{1}, \ldots, \alpha_{n}\right)$ of $B\left(\alpha_{1}, \ldots, \alpha_{n}\right)$ in $\mathcal{H} \cup \mathbb{C}$. It consists of the points $(z, \zeta) \in \mathcal{H} \cup \mathbb{C}$ satisfying all of the non-strict inequalities $|\mu z-\lambda|^{2}+|\mu|^{2} \zeta^{2} \geqslant 1$ that we have used to define $B\left(\alpha_{1}, \ldots, \alpha_{n}\right)$, and satisfy the additional condition that at least one of these non-strict inequalities is an equality. We will see below that $\partial B\left(\alpha_{1}, \ldots, \alpha_{n}\right)$ carries a natural cell structure. This, together with the following definitions, makes it possible to state the criterion which tells us when we have found all of the hemispheres relevant for the Bianchi fundamental polyhedron.

Definition 9. We shall say that the hemisphere $S_{\mu, \lambda}$ is strictly below the hemisphere $S_{\beta, \alpha}$ at a point $z \in \mathbb{C}$ if the following inequality is satisfied:

$$
\left|z-\frac{\alpha}{\beta}\right|^{2}-\frac{1}{|\beta|^{2}}<\left|z-\frac{\lambda}{\mu}\right|^{2}-\frac{1}{|\mu|^{2}} .
$$

This is, of course, an abuse of language because there may not be any points on $S_{\beta, \alpha}$ or $S_{\mu, \lambda}$ with coordinate $z$. However, if there is a point $(z, \zeta)$ on $S_{\mu, \lambda}$, the right-hand side of the inequality is just $-\zeta^{2}$. Thus, the left-hand side is negative and so of the form $-\left(\zeta^{\prime}\right)^{2}$. Clearly, $\left(z, \zeta^{\prime}\right) \in S_{\beta, \alpha}$ and $\zeta^{\prime}>\zeta$. We will further say that a point $(z, \zeta) \in \mathcal{H} \cup \mathbb{C}$ is strictly below a hemisphere $S_{\mu, \lambda}$, if there is a point $\left(z, \zeta^{\prime}\right) \in S_{\mu, \lambda}$ with $\zeta^{\prime}>\zeta$. 


\subsection{Singular points}

We call cusps the elements of the number field $K=\mathbb{Q}(\sqrt{-m})$ considered as points in the boundary of hyperbolic space, via an embedding $K \subset \mathbb{C} \cup\{\infty\} \cong \partial \mathcal{H}$. We write $\infty=1 / 0$, which we also consider as a cusp. It is well-known that the set of cusps is closed under the action of $\mathrm{SL}_{2}(\mathcal{O})$ on $\partial \mathcal{H}$ and that we have the following bijective correspondence between the $\mathrm{SL}_{2}(\mathcal{O})$ orbits of cusps and the ideal classes in $\mathcal{O}$. A cusp $\lambda / \mu$ is in the $\mathrm{SL}_{2}(\mathcal{O})$-orbit of the cusp $\lambda^{\prime} / \mu^{\prime}$, if and only if the ideals $\left(\lambda^{\prime}, \mu^{\prime}\right)$ and $(\lambda, \mu)$ are in the same ideal class. It immediately follows that the orbit of the cusp $\infty=1 / 0$ corresponds to the principal ideals. Let us call singular the cusps $\lambda / \mu$ such that $(\lambda, \mu)$ is not principal. And let us call singular points the singular cusps which lie in $\partial B$. It follows from the characterization of the singular points by Bianchi that they are precisely the points in $\mathbb{C} \subset \partial \mathcal{H}$ which cannot be strictly below any hemisphere. In the cases where $\mathcal{O}$ is a principal ideal domain, $K \cup\{\infty\}$ consists of only one $\mathrm{SL}_{2}(\mathcal{O})$-orbit, so there are no singular points. We use the following formulae derived by Swan, to compute representatives modulo the translations by $\Gamma_{\infty}$, of the singular points.

Lemma 10 (Swan [32]). The singular points of $K, \bmod \mathcal{O}$, are given by $p(r+\sqrt{-m}) / s$, where $p, r, s \in \mathbb{Z}, s>0,-s / 2<r \leqslant s / 2, s^{2} \leqslant r^{2}+m$ and:

- if $m \equiv 1$ or $2 \bmod 4, s \neq 1, s \mid r^{2}+m$, the numbers $p$ and $s$ are coprime, and $p$ is taken $\bmod s$;

- if $m \equiv 3 \bmod 4, s$ is even, $s \neq 2,2 s \mid r^{2}+m$, the numbers $p$ and $s / 2$ are coprime; $p$ is taken $\bmod s / 2$.

The singular points need not be considered in Swan's termination criterion, because they cannot be strictly below any hemisphere $S_{\mu, \lambda}$.

\subsection{Swan's termination criterion}

We observe that the set of $z \in \mathbb{C}$ over which some hemisphere is strictly below another is $\mathbb{C}$ or an open half-plane. In the latter case, the boundary of this is a line.

Notation 11. Denote by $L(\alpha / \beta, \lambda / \mu)$ the set of $z \in \mathbb{C}$ over which neither $S_{\beta, \alpha}$ is strictly below $S_{\mu, \lambda}$ nor vice versa.

This line is computed by turning the inequality in Definition 9 into an equation. Swan calls it the line over which the two hemispheres agree, and we will see later that the most important edges of the Bianchi fundamental polyhedron lie on the preimages of such lines. We now restrict our attention to a set of hemispheres which is finite modulo the translations in $\Gamma_{\infty}$. Consider a set of hemispheres $S\left(\alpha_{i}+\gamma\right)$, where the index $i$ runs from 1 through $n$, and $\gamma$ runs through $\mathcal{O}$. We call this set of hemispheres a collection, if every non-singular point $z \in \mathbb{C} \subset \partial \mathcal{H}$ is strictly below some hemisphere in our set. Now consider a set $B\left(\alpha_{1}, \ldots, \alpha_{n}\right)$ which is determined by such a collection of hemispheres.

Theorem 12 (Swan's termination criterion [32]). We have $B\left(\alpha_{1}, \ldots, \alpha_{n}\right)=B$ if and only if no vertex of $\partial B\left(\alpha_{1}, \ldots, \alpha_{n}\right)$ can be strictly below any hemisphere $S_{\mu, \lambda}$.

In other words, no vertex $v$ of $\partial B\left(\alpha_{1}, \ldots, \alpha_{n}\right)$ can lie strictly below any hemisphere $S_{\mu, \lambda}$. Let us call the coordinate $\zeta$ of the upper-half space model introduced at the beginning of $\S 2$ the height. With this criterion, it suffices to compute the cell structure of $\partial B\left(\alpha_{1}, \ldots, \alpha_{n}\right)$ to see whether our choice of hemispheres gives us the Bianchi fundamental polyhedron. This has only to be done modulo the translations of $\Gamma_{\infty}$, which preserve the height and, hence, the situations of being strictly below. Thus, our computations only need to be carried out on a finite set of hemispheres. 


\subsection{Computing the cell structure in the complex plane}

We will in a first step compute the image of the cell structure under the homeomorphism from $\partial B\left(\alpha_{1}, \ldots, \alpha_{n}\right)$ to $\mathbb{C}$ given by the vertical projection. For each 2-cell of this structure, there is an associated hemisphere $S_{\mu, \lambda}$. The interior of this 2-cell consists of the points $z \in \mathbb{C}$, where all other hemispheres in our collection are strictly below $S_{\mu, \lambda}$. Swan shows that this is the interior of a convex polygon. The edges of these polygons lie on real lines in $\mathbb{C}$ specified in Notation 11.

A vertex is an intersection point $z$ of any two of these lines involving the same hemisphere $S_{\mu, \lambda}$, if all other hemispheres in our collection are strictly below, or agree with, $S_{\mu, \lambda}$ at $z$.

\subsection{Lifting the cell structure back to hyperbolic space}

Now we can lift the cell structure back to $\partial B\left(\alpha_{1}, \ldots, \alpha_{n}\right)$, using the projection homeomorphism onto $\mathbb{C}$. The preimages of the convex polygons of the cell structure on $\mathbb{C}$, are totally geodesic hyperbolic polygons each lying on one of the hemispheres in our collection. These are the 2-cells of $\partial B\left(\alpha_{1}, \ldots, \alpha_{n}\right)$.

The edges of these hyperbolic polygons lie on the intersection arcs of pairs of hemispheres in our collection. As two Euclidean 2-spheres intersect, if they do so non-trivially, in a circle centred on the straight line which connects the two 2-sphere centres, such an intersection arc lies on a semicircle centred in the complex plane. The plane which contains this semicircle must be orthogonal to the connecting line, hence a vertical plane in $\mathcal{H}$. We can alternatively conclude the latter facts observing that an edge which two totally geodesic polygons have in common must be a geodesic segment. Lifting the vertices becomes now obvious from their definition. This enables us to check Swan's termination criterion.

We will now sketch Swan's proof of this criterion. Let $P$ be one of the convex polygons of the cell structure on $\mathbb{C}$. The preimage of $P$ lies on one hemisphere $S\left(\alpha_{i}\right)$ of our collection. Now the condition stated in Theorem 12 states that at the vertices of $P$, the hemisphere $S\left(\alpha_{i}\right)$ cannot be strictly below any other hemisphere. The points where $S\left(\alpha_{i}\right)$ can be strictly below some hemisphere constitute an open half-plane in $\mathbb{C}$, and hence cannot lie in the convex hull of the vertices of $P$, which is $P$. Theorem 12 now follows because $\mathbb{C}$ is tessellated by these convex polygons.

\section{Algorithms realizing Swan's concept}

From now on, we will work on putting Swan's concept into practice. We can reduce the set of hemispheres on which we carry out our computations, with the help of the following notion.

Definition 13. A hemisphere $S_{\mu, \lambda}$ is said to be everywhere below a hemisphere $S_{\beta, \alpha}$ when

$$
\left|\frac{\lambda}{\mu}-\frac{\alpha}{\beta}\right| \leqslant \frac{1}{|\beta|}-\frac{1}{|\mu|}
$$

Note that this is also the case when $S_{\mu, \lambda}=S_{\beta, \alpha}$. Any hemisphere which is everywhere below another one, does not contribute to the Bianchi fundamental polyhedron, in the following sense.

Proposition 14. Let $S\left(\alpha_{n}\right)$ be a hemisphere everywhere below some other hemisphere $S\left(\alpha_{i}\right)$, where $i \in\{1, \ldots, n-1\}$. Then $B\left(\alpha_{1}, \ldots, \alpha_{n}\right)=B\left(\alpha_{1}, \ldots, \alpha_{n-1}\right)$.

Proof. Write $\alpha_{n}=\lambda / \mu$ and $\alpha_{i}=\theta / \tau$ with $\lambda, \mu, \theta, \tau \in \mathcal{O}$. We take any point $(z, \zeta)$ strictly below $S_{\mu, \lambda}$ and show that it is also strictly below $S_{\tau, \theta}$. In terms of Notation 8 , this problem looks as follows: we assume that the inequality $|\mu z-\lambda|^{2}+|\mu|^{2} \zeta^{2}<1$ is satisfied, and show that this implies the inequality $|\tau z-\theta|^{2}+|\tau|^{2} \zeta^{2}<1$. The first inequality can be transformed into $|z-\lambda / \mu|^{2}+\zeta^{2}<1 /|\mu|^{2}$. Hence, $\sqrt{|z-\lambda / \mu|^{2}+\zeta^{2}}<1 /|\mu|$. We will insert this into the 
triangle inequality for the Euclidean distance in $\mathbb{C} \times \mathbb{R}$ applied to the three points $(z, \zeta)$, $(\lambda / \mu, 0)$ and $(\theta / \tau, 0)$, which is

$$
\sqrt{\left|z-\frac{\theta}{\tau}\right|^{2}+\zeta^{2}}<\left|\frac{\lambda}{\mu}-\frac{\theta}{\tau}\right|+\sqrt{\left|z-\frac{\lambda}{\mu}\right|^{2}+\zeta^{2}} .
$$

So we obtain $\sqrt{|z-\theta / \tau|^{2}+\zeta^{2}}<|\lambda / \mu-\theta / \tau|+1 /|\mu|$. By Definition 13, the expression on the right-hand side is smaller than or equal to $1 /|\tau|$. Therefore, we take the square and obtain $|z-\theta / \tau|^{2}+\zeta^{2}<1 /|\tau|^{2}$, which is equivalent to the claimed inequality.

Another notion that will be useful for our algorithm is as follows.

Definition 15. Let $z \in \mathbb{C}$ be a point lying within the vertical projection of $S_{\mu, \lambda}$. Define the lift on the hemisphere $S_{\mu, \lambda}$ of $z$ as the point on $S_{\mu, \lambda}$ the vertical projection of which is $z$.

Notation 16. Denote by the hemisphere list a list into which we will record a finite number of hemisphere s $S\left(\alpha_{1}\right), \ldots, S\left(\alpha_{n}\right)$. Its purpose is to determine a set $B\left(\alpha_{1}, \ldots, \alpha_{n}\right)$ in order to approximate, and finally obtain, the Bianchi fundamental polyhedron.

\subsection{The algorithm computing the Bianchi fundamental polyhedron}

We now describe the algorithm that we have realized using Swan's description; it is decomposed into Algorithms 1-3.

Initial step. We begin with the smallest value which the norm of a non-zero element $\mu \in \mathcal{O}$ can take, namely 1 . Then $\mu$ is a unit in $\mathcal{O}$, and for any $\lambda \in \mathcal{O}$, the pair $(\mu, \lambda)$ is unimodular. We can rewrite the fraction $\lambda / \mu$ such that $\mu=1$. We obtain the unit hemispheres (of radius 1 ), centred at the imaginary quadratic integers $\lambda \in \mathcal{O}$. We record into the hemisphere list those which touch the Bianchi fundamental polyhedron, i.e. those the centre of which lies in the fundamental rectangle $D_{0}$ (of Notation 4 ) for the action of $\Gamma_{\infty}$ on the complex plane.

Step $A$. Increase $|\mu|$ to the next higher value which the norm takes on elements of $\mathcal{O}$. Run through all of the finitely many $\mu$ which have this norm. For each of these $\mu$, run through all of the finitely many $\lambda$ with $\lambda / \mu$ in the fundamental rectangle $D_{0}$. Check that $(\mu, \lambda)=\mathcal{O}$ and that the hemisphere $S_{\mu, \lambda}$ is not everywhere below a hemisphere $S_{\beta, \alpha}$ in the hemisphere list. If these two checks are passed, record $(\mu, \lambda)$ into the hemisphere list.

We repeat Step A until $|\mu|$ has reached an expected value. Then we check whether we have found all of the hemispheres which touch the Bianchi fundamental polyhedron, as follows.

Step $B$. We compute the lines $L(\alpha / \beta, \lambda / \mu)$ of Notation 11, over which two hemispheres agree, for all pairs $S_{\beta, \alpha}, S_{\mu, \lambda}$ in the hemisphere list which touch one another.

Then, for each hemisphere $S_{\beta, \alpha}$, we compute the intersection points of each two lines $L(\alpha / \beta, \lambda / \mu)$ and $L(\alpha / \beta, \theta / \tau)$ referring to $\alpha / \beta$.

We drop the intersection points at which $S_{\beta, \alpha}$ is strictly below some hemisphere in the list.

We erase the hemispheres from our list, for which less than three intersection points remain. We can do this because a hemisphere which touches the Bianchi fundamental polyhedron only in two vertices shares only an edge with it and no 2-cell.

Now, the vertices of $B\left(\alpha_{1}, \ldots, \alpha_{n}\right) \cap D_{\infty}$ are the lifts of the remaining intersection points. Thus, we can check Swan's termination criterion (Theorem 12), which we do as follows. We pick the lowest value $\zeta>0$ for which $(z, \zeta) \in \mathcal{H}$ is the lift inside hyperbolic space of a remaining intersection point $z$. 
If $\zeta \geqslant 1 /|\mu|$, then all (infinitely many) remaining hemispheres have radius at most $\zeta$, so $(z, \zeta)$ cannot be strictly below them. So Swan's termination criterion is fulfilled, we have found the Bianchi fundamental polyhedron, and can proceed by determining its cell structure.

Otherwise, $\zeta$ becomes the new expected value for $1 /|\mu|$. We repeat Step A until $|\mu|$ reaches $1 / \zeta$ and then proceed again with Step B.

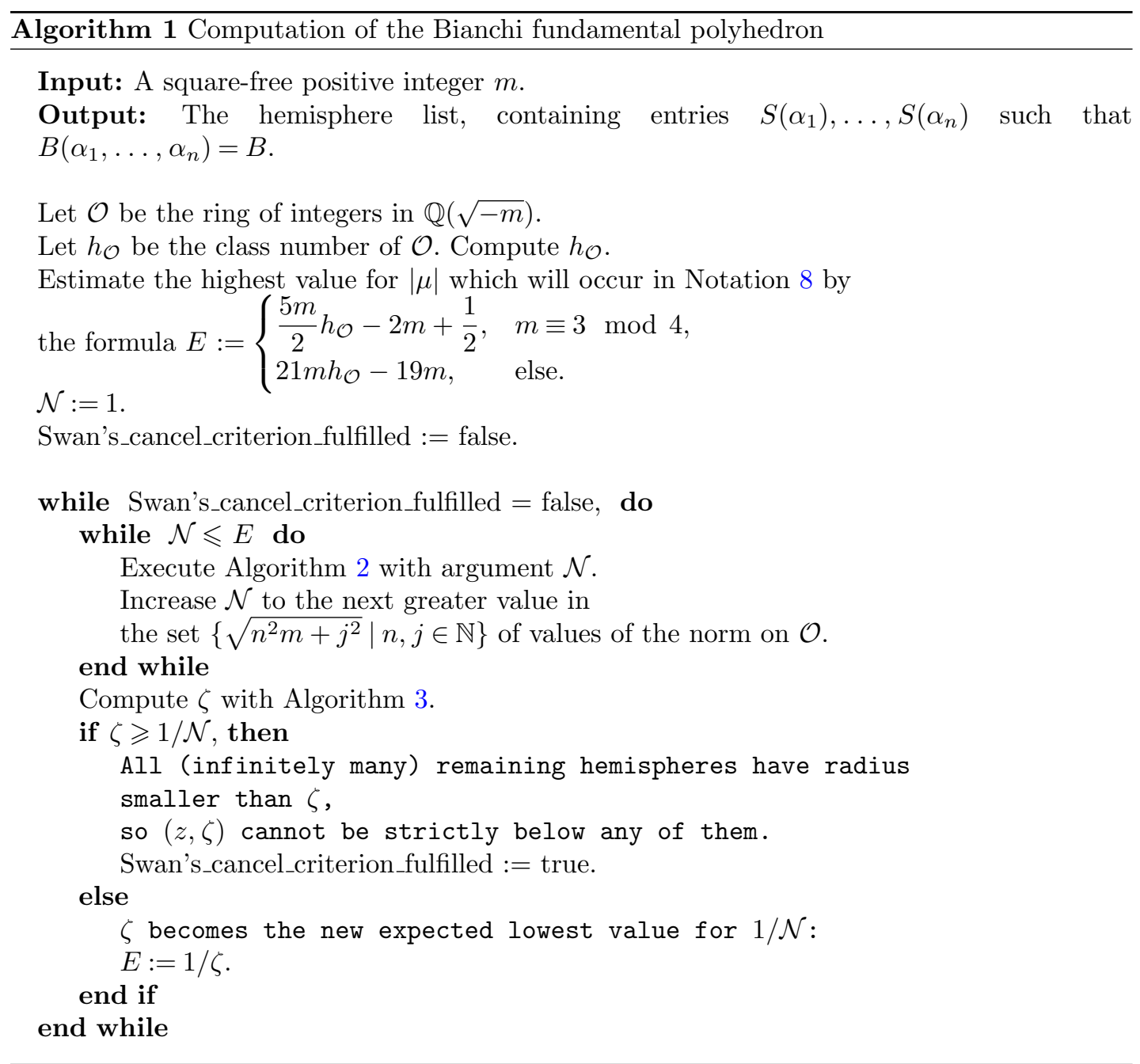

Proposition 17. The hemisphere list, as computed by Algorithm 1, determines the Bianchi fundamental polyhedron. This algorithm terminates within finite time.

Proof. The proof proceeds via the following steps.

- The value $\zeta$ is the minimal height of the non-singular vertices of the cell complex $\partial B\left(\alpha_{1}, \ldots, \alpha_{n}\right)$ determined by the hemisphere list $\left\{S\left(\alpha_{1}\right), \ldots, S\left(\alpha_{n}\right)\right\}$.

All of the hemispheres which are not in the list have radius smaller than $1 / \mathcal{N}$. By Remark 19, the inequality $\zeta \geqslant 1 / \mathcal{N}$ will become satisfied; and then no nonsingular vertex of $\partial B\left(\alpha_{1}, \ldots, \alpha_{n}\right)$ can be strictly below any of them. Hence, by Theorem 12, $B\left(\alpha_{1}, \ldots, \alpha_{n}\right)=B$ and we obtain the Bianchi fundamental polyhedron as $B\left(\alpha_{1}, \ldots, \alpha_{n}\right) \cap D_{\infty}$. 
- We now consider the run-time. By Theorem 6, the set of hemispheres

$$
\left\{S_{\mu, \lambda} \mid S_{\mu, \lambda} \text { touches the Bianchi fundamental polyhedron }\right\}
$$

is finite. So, there exists an $S_{\mu, \lambda}$ for which the norm of $\mu$ takes its maximum on this finite set. The variable $\mathcal{N}$ reaches this maximum for $|\mu|$ after a finite number of steps and then Swan's termination criterion is fulfilled. The latter steps require a finite run-time because of Propositions 20 and 21.

Swan explains furthermore how to obtain an a priori bound for the norm of the $\mu \in \mathcal{O}$ occurring for such hemispheres $S_{\mu, \lambda}$, but he states that this upper bound for $|\mu|$ is much too large. So instead of the theory behind Theorem 6, we use Swan's termination criterion (Theorem 12 above) to limit the number of steps in our computations. We then obtain the following observation.

Observation 18. We can give bounds for $|\mu|$ in the cases where $K$ is of class number 1 or 2 (there are 9 cases of class number 1 and 18 cases of class number 2, and we have performed the computation for all of them). They are as follows:

$$
\begin{cases}K \text { of class number } 1: & |\mu| \leqslant \frac{|\Delta|+1}{2}, \\ K \text { of class number } 2: \begin{cases}|\mu| \leqslant 3|\Delta| & m \equiv 3 \bmod 4, \\ |\mu| \leqslant\left(5+\frac{61}{116}\right)|\Delta|, & \text { otherwise, }\end{cases} \end{cases}
$$

where $\Delta$ is the discriminant of

$$
K=\mathbb{Q}(\sqrt{-m}) \quad \text { i.e. }|\Delta|= \begin{cases}m, & m \equiv 3 \bmod 4, \\ 4 m & \text { otherwise. }\end{cases}
$$

Remark 19. In Algorithm 1, we have chosen the value $E$ by an extrapolation formula for Observation 18. If this is greater than the exact bound for $|\mu|$, the algorithm computes additional hemispheres which do not contribute to the Bianchi fundamental polyhedron. On the other hand, if $E$ is smaller than the exact bound for $|\mu|$, it will be increased in the outer while loop of the algorithm, until it is sufficiently large. Then the algorithm performs some preliminary computations of the intersection lines and vertices, which cost additional run-time. Thus, our extrapolation formula is aimed at choosing $E$ slightly greater than the exact bound for $|\mu|$ we expect.

Proposition 20. Algorithm 2 finds all of the hemispheres of radius $1 / \mathcal{N}$, on which a 2 -cell of the Bianchi fundamental polyhedron can lie. This algorithm terminates within finite time.

Proof. The proof proceeds with two steps.

- Directly from the definition of the hemispheres $S_{\mu, \lambda}$, it follows that the radius is given by $1 /|\mu|$. So our algorithm runs through all $\mu$ in question. By construction of the Bianchi fundamental polyhedron $D$, the hemispheres on which a 2-cell of $D$ lies must have their centre in the fundamental rectangle $D_{0}$. By Proposition 14, such hemispheres cannot be everywhere below some other hemisphere in the list.

- Now we consider the run-time of the algorithm. There are finitely many $\mu \in \mathcal{O}$ the norm of which takes a given value. For a given $\mu$, there are finitely many $\lambda \in \mathcal{O}$ such that $\lambda / \mu$ is in the fundamental rectangle $D_{0}$. Therefore, this algorithm consists of finite loops and terminates within finite time. 


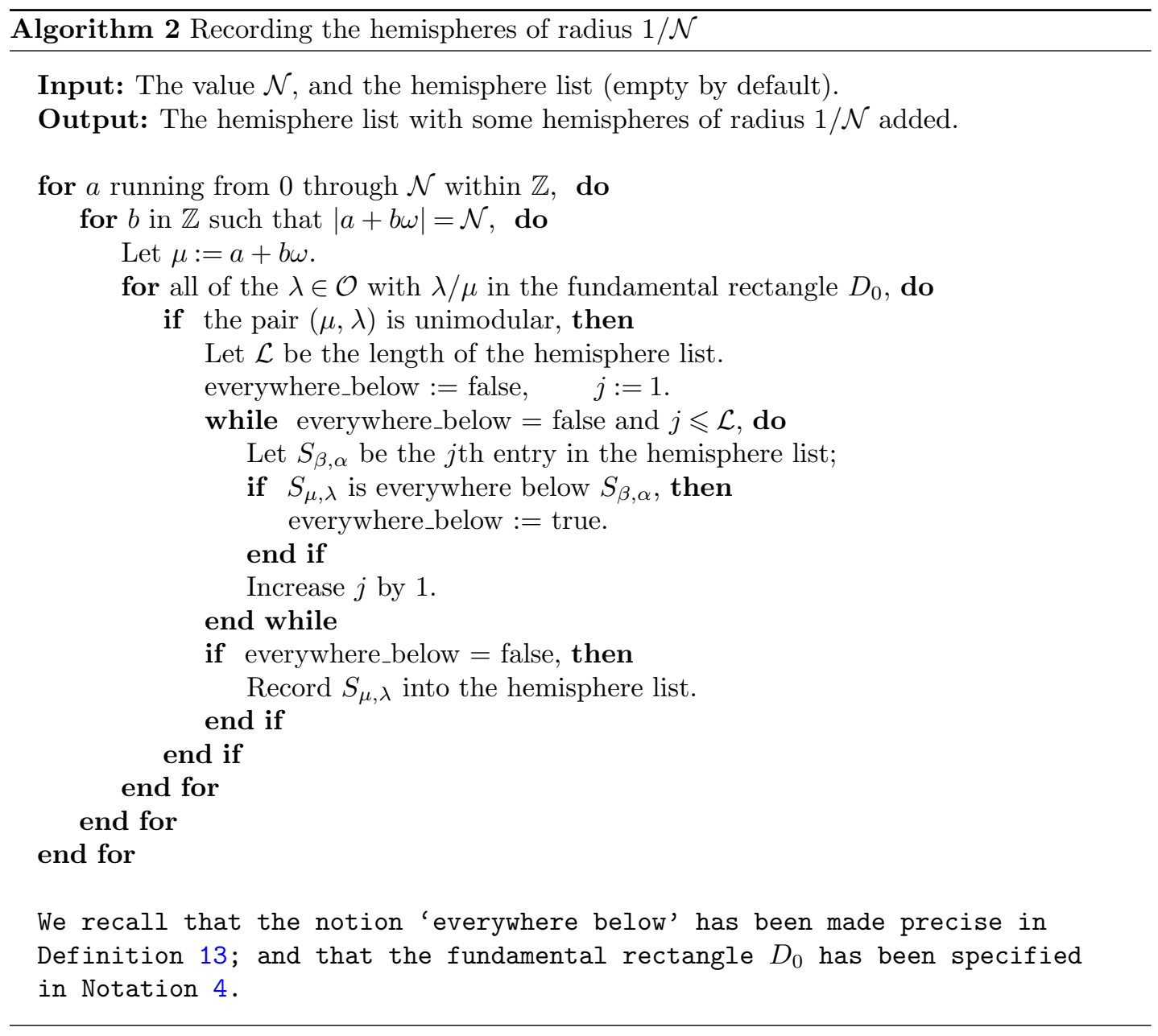

Proposition 21. Algorithm 3 finds the minimal height occurring amongst the non-singular vertices of $\partial B\left(\alpha_{1}, \ldots, \alpha_{n}\right)$. This algorithm erases only such hemispheres from the list, which do not change $\partial B\left(\alpha_{1}, \ldots, \alpha_{n}\right)$. It terminates within finite time.

Proof. The proof proceeds via the following steps.

- The heights of the points in $\mathcal{H}$ are preserved by the action of the translation group $\Gamma_{\infty}$, so we only need to consider representatives in the fundamental domain $D_{\infty}$ for this action. Our algorithm computes the entire cell structure of $\partial B\left(\alpha_{1}, \ldots, \alpha_{n}\right) \cap D_{\infty}$, as described in $\S 6.4$. The number of lines to intersect is smaller than the square of the length of the hemisphere list, and thus finite. As a consequence, the minimum of the height has to be taken only on a finite set of intersection points, whence the first claim.

- If a cell of $\partial B\left(\alpha_{1}, \ldots, \alpha_{n}\right)$ lies on a hemisphere, then its vertices are lifts of intersection points. So we can erase the hemispheres which are strictly below some other hemispheres at all of the intersection points, without changing $\partial B\left(\alpha_{1}, \ldots, \alpha_{n}\right)$.

- Now we consider the run-time. This algorithm consists of loops running through the hemisphere list, which has finite length. Within one of these loops, there is a loop running through the set of pairs of lines $L(\alpha / \beta, \lambda / \mu)$. A (far too large) bound for the cardinality of this set is given by the fourth power of the length of the hemisphere list. The steps performed within these loops are very delimited and easily seen to be of finite run-time. 


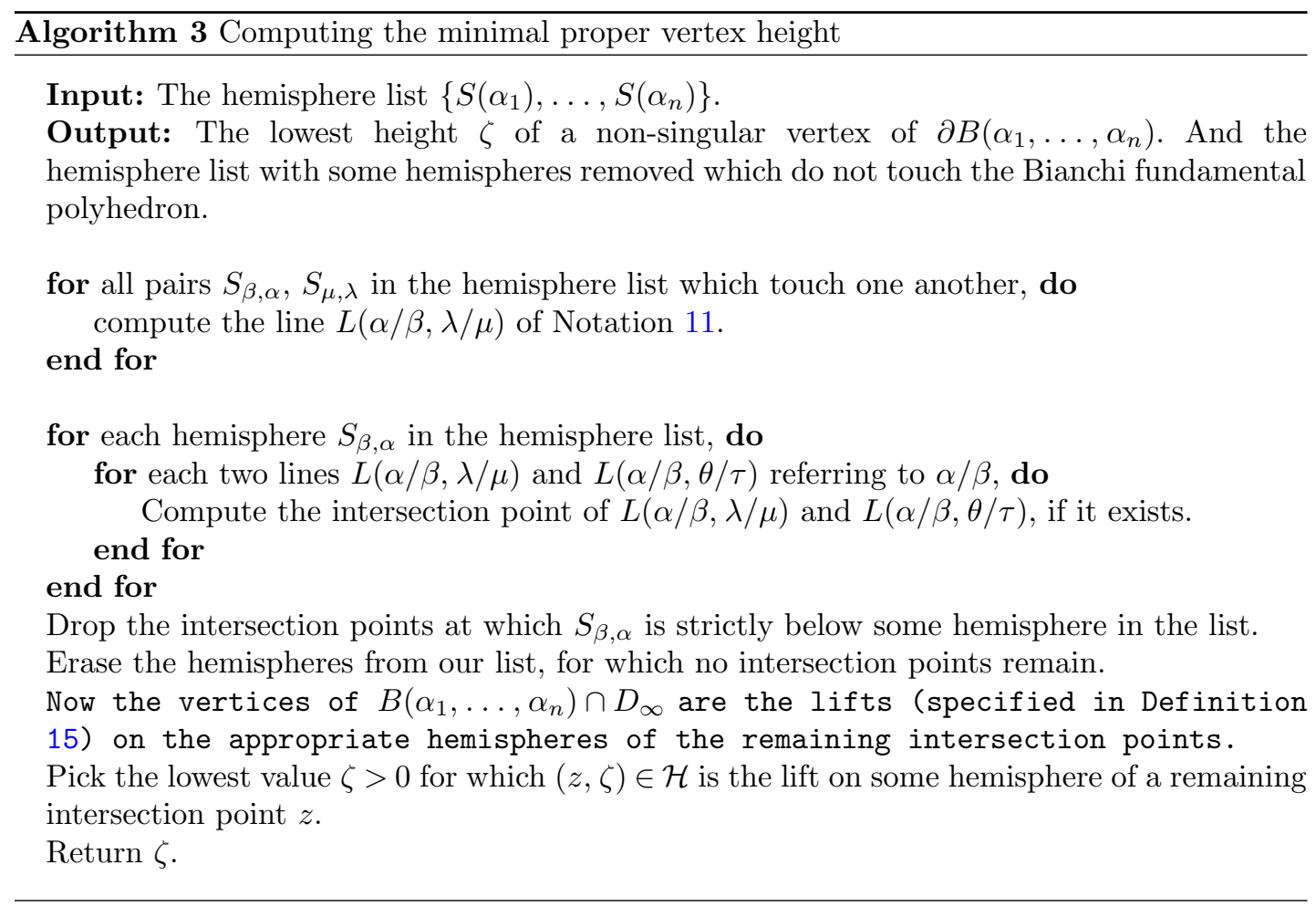

\subsection{The cell complex and its orbit space}

With the method described in $\S 6.4$, we obtain a cell structure on the boundary of the Bianchi fundamental polyhedron. The cells in this structure which touch the cusp $\infty$ are easily determined: they are four 2-cells each lying on one of the Euclidean vertical planes bounding the fundamental domain $D_{\infty}$ for $\Gamma_{\infty}$ specified in Notation 4 ; and four 1-cells each lying on one of the intersection lines of these planes. The other 2-cells in this structure lie each on one of the hemispheres determined with our realization of Swan's algorithm.

As the Bianchi fundamental polyhedron is a hyperbolic polyhedron up to some missing cusps, its boundary cells can be oriented as its facets. Once the cell structure is subdivided until the cells are fixed pointwise by their stabilizers, this cell structure with orientation is transported onto the whole hyperbolic space by the action of $\Gamma$.

\subsection{Computing the vertex stabilizers and identifications}

Let us state explicitly the $\Gamma$-action on the upper-half space model $\mathcal{H}$, in the form in which we will use it rather than in its historical form.

Lemma 22 (Poincaré). If $\gamma=\left(\begin{array}{ll}a & b \\ c & d\end{array}\right) \in \mathrm{GL}_{2}(\mathbb{C})$, the action of $\gamma$ on $\mathcal{H}$ is given by $\gamma \cdot(z, \zeta)=$ $\left(z^{\prime}, \zeta^{\prime}\right)$, where

$$
\zeta^{\prime}=\frac{|\operatorname{det} \gamma| \zeta}{|c z-d|^{2}+\zeta^{2}|c|^{2}}, \quad z^{\prime}=\frac{(\overline{d-c z})(a z-b)-\zeta^{2} \bar{c} a}{|c z-d|^{2}+\zeta^{2}|c|^{2}}
$$

From this operation formula, we establish equations and inequalities on the entries of a matrix sending a given point $(z, \zeta)$ to another given point $\left(z^{\prime}, \zeta^{\prime}\right)$ in $\mathcal{H}$. We will use them in Algorithm 4 to compute such matrices. For the computation of the vertex stabilizers, 
we have $(z, \zeta)=\left(z^{\prime}, \zeta^{\prime}\right)$, which simplifies the below equations and inequalities as well as the pertinent algorithm. First, we fix a basis for $\mathcal{O}$ as the elements 1 and

$$
\omega:= \begin{cases}\sqrt{-m}, & m \equiv 1 \text { or } 2 \bmod 4, \\ -\frac{1}{2}+\frac{1}{2} \sqrt{-m}, & m \equiv 3 \bmod 4 .\end{cases}
$$

As we have put $m \neq 1$ and $m \neq 3$, the only units in the $\operatorname{ring} \mathcal{O}$ are \pm 1 . We will use the notation $\lceil x\rceil:=\min \{n \in \mathbb{Z} \mid n \geqslant x\}$ and $\lfloor x\rfloor:=\max \{n \in \mathbb{Z} \mid n \leqslant x\}$ for $x \in \mathbb{R}$.

Lemma 23. Let $m \equiv 3 \bmod 4$. Let $\left(\begin{array}{ll}a & b \\ c & d\end{array}\right) \in \mathrm{SL}_{2}(\mathcal{O})$ be a matrix sending $(z, r)$ to $(\zeta, \rho) \in \mathcal{H}$. Write $c$ in the basis as $j+k \omega$, where $j, k \in \mathbb{Z}$. Then $|c|^{2} \leqslant 1 / r \rho,|j| \leqslant \sqrt{(1+1 / m) / r \rho}$ and

$$
\frac{2 j}{m+1}-2 \frac{\sqrt{((m+1) / r \rho)-j^{2} m}}{m+1} \leqslant k \leqslant \frac{2 j}{m+1}+2 \frac{\sqrt{((m+1) / r \rho)-j^{2} m}}{m+1} .
$$

Proof. From the operation equation $\left(\begin{array}{ll}a & b \\ c & d\end{array}\right) \cdot(z, r)=(\zeta, \rho)$, we deduce $|c z-d|^{2}+r^{2}|c|^{2}=r / \rho$ and conclude $r^{2}|c|^{2} \leqslant r / \rho$, whence the first inequality. We insert $|c|^{2}=(j-k / 2)^{2}+m(k / 2)^{2}$ $=j^{2}+m+1 / 4 k^{2}-j k$ into it, and obtain

$$
0 \geqslant k^{2}-\frac{4 j}{m+1} k+\frac{4}{m+1}\left(j^{2}-\frac{1}{r \rho}\right)=: f(k) .
$$

We observe that $f(k)$ is a quadratic function in $k \in \mathbb{Z} \subset \mathbb{R}$, taking its values exclusively in $\mathbb{R}$. Hence, its graph has the shape of a parabola, and the negative values of $f(k)$ appear exactly on the interval where $k$ is between its two zeros,

$$
k_{ \pm}=\frac{2 j}{m+1} \pm 2 \frac{\sqrt{\Delta}}{m+1} \quad \text { where } \Delta=\frac{m+1}{r \rho}-j^{2} m .
$$

This implies the third and fourth claimed inequalities. As $k$ is a real number, $\Delta$ must be nonnegative in order that $f(k)$ be non-positive. Hence, $j^{2} \leqslant(1+1 / m) / r \rho$, which gives the second claimed inequality.

Lemma 24. Under the assumptions of Lemma 23, write $d$ in the basis as $q+s \omega$, where $q, s \in \mathbb{Z}$. Write $c z$ in the basis as $R(c z)+W(c z) \omega$, where $R(c z), W(c z) \in \mathbb{Q}$. Then

and

$$
W(c z)-2 \sqrt{\frac{(r / \rho)-r^{2}|c|^{2}}{m}} \leqslant s \leqslant W(c z)+2 \sqrt{\frac{(r / \rho)-r^{2}|c|^{2}}{m}},
$$

$$
q=R(c z)-\frac{W(c z)}{2}+\frac{s}{2} \pm \sqrt{\frac{r}{\rho}-r^{2}|c|^{2}-m\left(\frac{W(c z)}{2}-\frac{s}{2}\right)^{2}} .
$$

Proof. Recall that $\omega=-\frac{1}{2}+\frac{1}{2} \sqrt{-m}$, so $\overline{q+s \omega}=q-s / 2-s / 2 \sqrt{-m}$. The operation equation yields $|c z-d|^{2}+r^{2}|c|^{2}=r / \rho$. From this, we derive

$$
\begin{aligned}
\frac{r}{\rho}-r^{2}|c|^{2} & =(c z-(q+s \omega))\left(\overline{c z}-\left(q-\frac{s}{2}-\frac{s}{2} \sqrt{-m}\right)\right) \\
& =\left(\operatorname{Re}(c z)-q+\frac{s}{2}\right)^{2}+\left(\operatorname{Im}(c z)-\frac{s}{2} \sqrt{m}\right)^{2} \\
& =\operatorname{Re}(c z)^{2}+q^{2}-q s+\frac{s^{2}}{4}-2 \operatorname{Re}(c z) q+\operatorname{Re}(c z) s+\left(\operatorname{Im}(c z)-\frac{s}{2} \sqrt{m}\right)^{2} .
\end{aligned}
$$

We solve for $q$,

$$
q^{2}+(-2 \operatorname{Re}(c z)-s) q+\left(\operatorname{Re}(c z)+\frac{s}{2}\right)^{2}+\left(\operatorname{Im}(c z)-\frac{s}{2} \sqrt{m}\right)^{2}-\frac{r}{\rho}+r^{2}|c|^{2}=0
$$




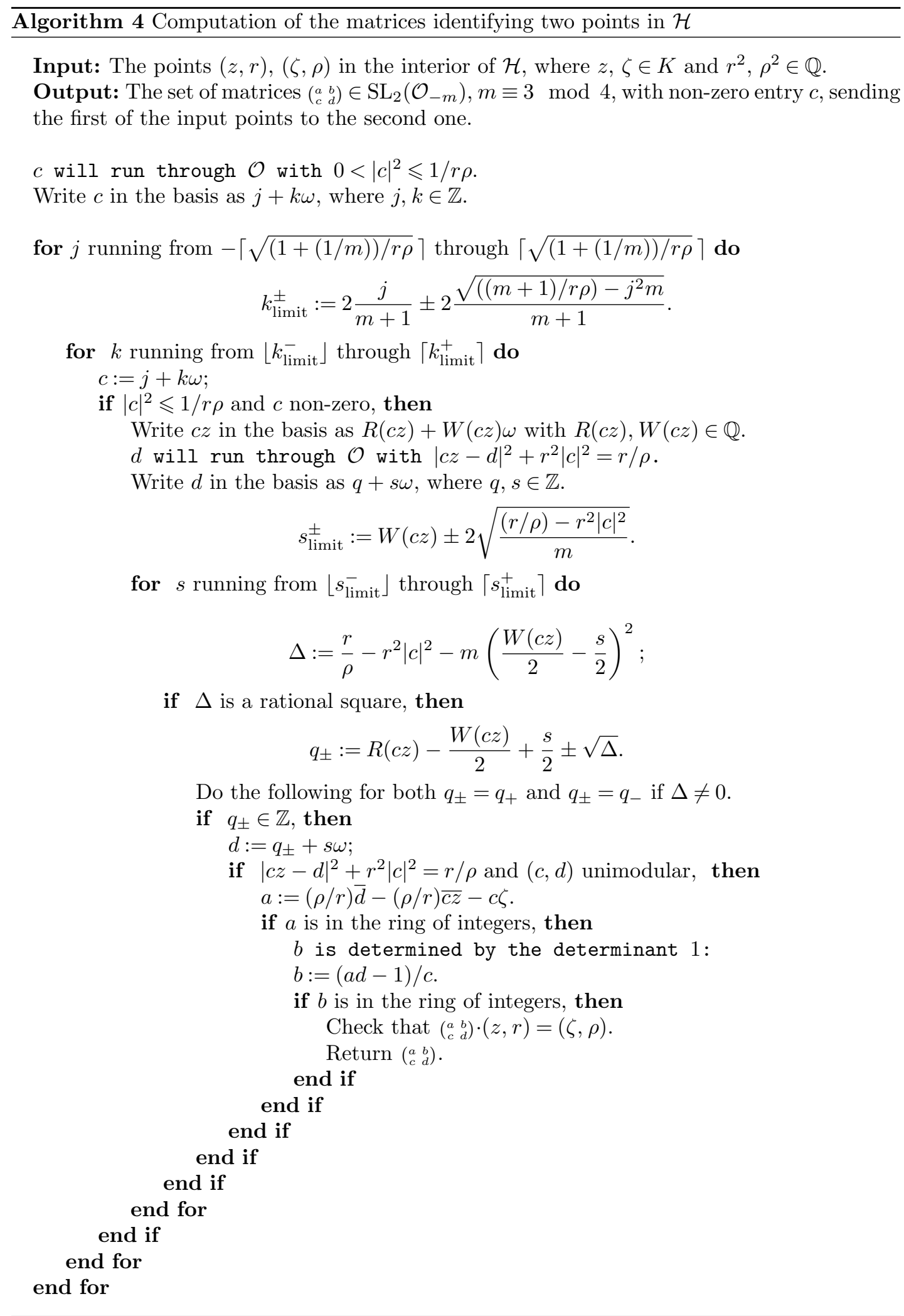


and find

$$
q_{ \pm}=\operatorname{Re}(c z)+\frac{s}{2} \pm \sqrt{\Delta} \quad \text { where } \Delta=\frac{r}{\rho}-r^{2}|c|^{2}-\left(\operatorname{Im}(c z)-\frac{s}{2} \sqrt{m}\right)^{2} .
$$

We express this as

$$
q_{ \pm}=R(c z)-\frac{W(c z)}{2}+\frac{s}{2} \pm \sqrt{\Delta} \quad \text { where } \Delta=\frac{r}{\rho}-r^{2}|c|^{2}-m\left(\frac{W(c z)}{2}-\frac{s}{2}\right)^{2}
$$

which is the claimed equation. The condition that $q$ must be a rational integer implies $\Delta \geqslant 0$, which can be rewritten in the claimed inequalities.

We further state a simple inequality in order to prove that Algorithm 4 terminates in finite time.

Lemma 25. Let $K=\mathbb{Q}(\sqrt{-m})$ with $m \neq 3$. Let $c, z \in K$. Write their product $c z$ in the $\mathbb{Q}$-basis $\{1, \omega\}$ for $K$ as $R(c z)+W(c z) \omega$. Then the inequality $|W(c z)| \leqslant|c| \cdot|z|$ holds.

Proof. Let $x+y \omega \in K$ with $x, y \in \mathbb{Q}$. Our first step is to show that $|y| \leqslant|x+y \omega|$. Consider the case $m \equiv 1$ or $2 \bmod 4$. Then

$$
|x+y \omega|=\sqrt{x^{2}+m y^{2}} \geqslant \sqrt{m}|y| \geqslant|y|,
$$

and we have shown our claim. Else consider the case $m \equiv 3 \bmod 4$. Then,

$$
|x+y \omega|=\sqrt{(x+\omega y)(x+\bar{\omega} y)}=\sqrt{\left(x^{2}-2 x \frac{y}{2}+\frac{y^{2}}{4}\right)+\frac{m}{4} y^{2}} \geqslant \frac{\sqrt{m}}{2}|y|,
$$

and our claim follows for $m>3$. Now we have shown that $|W(c z)| \leqslant|c z|$ and we use some embedding of $K$ into $\mathbb{C}$ to verify the equation $|c z|=|c| \cdot|z|$.

Proposition 26. Let $m \equiv 3 \bmod 4$. Then Algorithm 4 gives all of the matrices $\left(\begin{array}{ll}a & b \\ c & d\end{array}\right) \in$ $\mathrm{SL}_{2}(\mathcal{O})$ with $c \neq 0$, sending $(z, r)$ to $(\zeta, \rho) \in \mathcal{H}$. It terminates in finite time.

Proof. The proof proceeds via the following steps.

- The first claim is easily established using the bounds and formulae stated in Lemmas 23 and 24.

- Now we consider the run-time. This algorithm consists of three loops the limits of which are at most linear expressions in $1 / \sqrt{r \rho}$. For $s_{\text {limit }}^{ \pm}$, we use Lemma 25 and $r^{2}|c|^{2} \leqslant r / \rho$ to see this (we obtain a factor $|z|$ here, which we can neglect).

Finally, it should be said that the scope of computations one can do with geometric models for the Bianchi groups does not stop once the integral homology of the full group is known. There is further interest in homology with twisted coefficients, congruence subgroups and modular forms (see, for instance, $[\mathbf{2 8}, \mathbf{3 0}]$ ). Currently, Page [16] is working on optimizing algorithms in order to obtain more cell complexes for Bianchi groups and other Kleinian groups.

Acknowledgements. The author would like to thank Bill Allombert (PARI/GP Development Headquarters) and Philippe Elbaz-Vincent (UJF Grenoble) for invaluable help with the development of Bianchi.gp. He is grateful to the Weizmann Institute of Science for providing him with access to its high-performance computation clusters in order to establish the cell complexes database; and to Stephen S. Gelbart and Graham Ellis for support and encouragement. 


\section{References}

1. M. T. AranÉs, 'Modular symbols over number fields', PhD Thesis, University of Warwick, 2010.

2. L. BiAnCHI, 'Sui gruppi di sostituzioni lineari con coefficienti appartenenti a corpi quadratici immaginarî', Math. Ann. 40 (1892) no. 3, 332-412.

3. N. Bergeron and A. Venkatesh, 'The asymptotic growth of torsion homology for arithmetic groups', J. Inst. Math. Jussieu (2012), to appear, doi: 10.1017/S1474748012000667.

4. K. S. Brown, Cohomology of groups, Graduate Texts in Mathematics, 87 (Springer, New York, 1982).

5. J. BYGOTT, 'Modular forms and modular symbols over imaginary quadratic fields', PhD Thesis, University of Exeter, 1998.

6. J. E. Cremona and M. P. Lingham, 'Finding all elliptic curves with good reduction outside a given set of primes', Experiment. Math. 16 (2007) no. 3, 303-312; MR 2367320(2008k:11057).

7. J. E. Cremona, 'Hyperbolic tessellations, modular symbols, and elliptic curves over complex quadratic fields', Compositio Math. 51 (1984) no. 3, 275-324.

8. J. Elstrodt, F. Grunewald and J. Mennicke, Groups acting on hyperbolic space, Springer Monographs in Mathematics (Springer, Berlin, 1998).

9. B. Fine, Algebraic theory of the Bianchi groups, Monographs and Textbooks in Pure and Applied Mathematics, 129 (Marcel Dekker Inc., New York, 1989).

10. D. FlöGE, 'Zur Struktur der $\mathrm{PSL}_{2}$ über einigen imaginär-quadratischen Zahlringen', Dissertation, Johann-Wolfgang-Goethe-Universität, Fachbereich Mathematik, 1980.

11. D. FlÖGE, 'Zur Struktur der $\mathrm{PSL}_{2}$ über einigen imaginär-quadratischen Zahlringen', Math. Z. 183 (1983) no. 2, 255-279.

12. G. Humbert, 'Sur la réduction des formes d'Hermite dans un corps quadratique imaginaire', C. R. Acad. Sci. Paris 16 (1915) 189-196.

13. M. Lingham, 'Modular forms and elliptic curves over imaginary quadratic fields', PhD Thesis, University of Nottingham, 2005.

14. C. Maclachlan and A. W. Reid, The arithmetic of hyperbolic 3-manifolds, Graduate Texts in Mathematics, 219 (Springer-Verlag, New York, 2003).

15. E. R. MendozA, 'Cohomology of $\mathrm{PGL}_{2}$ over imaginary quadratic integers', Bonner Mathematische Schriften, 128 (Dissertation, Rheinische Friedrich-Wilhelms-Universität, Mathematisches Institut, 1979).

16. A. PAGE, 'Computing arithmetic Kleinian groups', Preprint, 2012 , http://hal.archives-ouvertes.fr/hal-00703043.

17. H. Poincaré, 'Mémoire: Les groupes Kleinéens', Acta Math. 3 (1883) no. 1, 49-92.

18. A. D. RAhm, Bianchi.gp, Open source program (GNU general public license), validated by the CNRS: http://www.projet-plume.org/fiche/bianchigp, subject to the Certificat de Compétences en Calcul Intensif (C3I) and part of the GP scripts library of Pari/GP Development Center, 2010.

19. A. D. Rahm, 'Homology and K-theory of the Bianchi groups', C. R. Math. Acad. Sci. Paris 349 (2011) no. 11-12, 615-619; MR 2817377(2012e:20116).

20. A. D. RAHM, 'The homological torsion of $\mathrm{PSL}_{2}$ of the imaginary quadratic integers', Trans. Amer. Math. Soc. 365 (2013) no. 3, 1603-1635; MR 3003276.

21. A. D. RAHM, 'Accessing the Farrell-Tate cohomology of discrete groups', Preprint, 2012, http://hal.archives-ouvertes.fr/hal-00618167.

22. A. D. Rahm, 'On a question of Serre', C. R. Math. Acad. Sci. Paris 350 (2012) no. 15-16, 741-744; MR 2981344.

23. A. D. RAHM and M. FuChS, 'The integral homology of $\mathrm{PSL}_{2}$ of imaginary quadratic integers with non-trivial class group', J. Pure Appl. Algebra 215 (2011) no. 6, 1443-1472; MR 2769243.

24. A. D. RAhm and M. H. ŞEngün, 'On level one cuspidal Bianchi modular forms', LMS J. Comput. Math. 16 (2013) 187-199.

25. R. RILEY, 'Applications of a computer implementation of Poincaré's theorem on fundamental polyhedra', Math. Comp. 40 (1983) no. 162, 607-632; MR 689477(85b:20064).

26. A. Scheutzow, 'Computing rational cohomology and Hecke eigenvalues for Bianchi groups', J. Number Theory 40 (1992) no. 3, 317-328.

27. J. Schwermer and K. Vogtmann, 'The integral homology of $\mathrm{SL}_{2}$ and $\mathrm{PSL}_{2}$ of Euclidean imaginary quadratic integers', Comment. Math. Helv. 58 (1983) no. 4, 573-598.

28. M. H. ŞENGüN, 'On the integral cohomology of Bianchi groups', Experiment. Math. 20 (2011) no. 4, 487-505; MR 2859903.

29. M. H. ŞEnGÜN, 'Arithmetic aspects of Bianchi groups', Preprint, 2012, arXiv:1204.6697.

30. M. H. ŞENGÜN and S. TURKELLI, 'Weight reduction for mod $\ell$ Bianchi modular forms', J. Number Theory 129 (2009) no. 8, 2010-2019; MR 2522720(2010c:11064).

31. J.-P. Serre, 'Le problème des groupes de congruence pour SL(2)', Ann. of Math. (2) 92 (1970) $489-527$.

32. R. G. SwAn, 'Generators and relations for certain special linear groups', Adv. Math. 6 (1971) 1-77.

33. K. Vogtmann, 'Rational homology of Bianchi groups', Math. Ann. 272 (1985) no. 3, 399-419.

34. E. WhitLey, 'Modular symbols and elliptic curves over imaginary quadratic fields', PhD Thesis, University of Exeter, 1990.

35. D. YASAKI, 'Hyperbolic tessellations associated to Bianchi groups', Proceedings 9th International Symposium on Algorithmic Number Theory (ANTS-IX), Nancy, France, 19-23 July 2010, 385-396. MR 2721434(2012g:11069). 
Alexander D. Rahm

Department of Mathematics,

National University of Ireland at Galway

Ireland

Alexander.Rahm@nuigalway.ie 